\title{
Sleep Loss Drives Brain Region-Specific and Cell Type- Specific Alterations in Ribosome-Associated Transcripts Involved in Synaptic Plasticity and Cellular Timekeeping
}

\author{
Carlos Puentes-Mestril, James Delorme, ${ }^{\circledR}$ Lijing Wang, Marcus Donnelly, Donald Popke, Sha Jiang, and \\ Sara J. Aton \\ Department of Molecular, Cellular, and Developmental Biology, University of Michigan, Ann Arbor, Michigan 48019
}

Sleep and sleep loss are thought to impact synaptic plasticity, and recent studies have shown that sleep and sleep deprivation (SD) differentially affect gene transcription and protein translation in the mammalian forebrain. However, much less is known regarding how sleep and SD affect these processes in different microcircuit elements within the hippocampus and neocortex, for example, in inhibitory versus excitatory neurons. Here, we use translating ribosome affinity purification (TRAP) and in situ hybridization to characterize the effects of sleep versus SD on abundance of ribosome-associated transcripts in Camk2a-expressing $\left(\right.$ Camk2a $\left.{ }^{+}\right)$pyramidal neurons and parvalbumin-expressing $\left(\mathrm{PV}^{+}\right)$interneurons in the hippocampus and neocortex of male mice. We find that while both Camk2a ${ }^{+}$neurons and $\mathrm{PV}^{+}$interneurons in neocortex show concurrent SD-driven increases in ribosome-associated transcripts for activity-regulated effectors of plasticity and transcriptional regulation, these transcripts are minimally affected by SD in hippocampus. Similarly, we find that while SD alters several ribosome-associated transcripts involved in cellular timekeeping in neocortical Camk2a ${ }^{+}$and $\mathrm{PV}^{+}$neurons, effects on circadian clock transcripts in hippocampus are minimal, and restricted to Camk2a ${ }^{+}$neurons. Taken together, our results indicate that SD effects on transcripts associated with translating ribosomes are both cell type-specific and brain region-specific, and that these effects are substantially more pronounced in the neocortex than the hippocampus. We conclude that SD-driven alterations in the strength of synapses, excitatory-inhibitory (E-I) balance, and cellular timekeeping are likely more heterogeneous than previously appreciated.

Key words: circadian rhythms; interneuron; plasticity; principal neurons; sleep deprivation; translation

Significance Statement

Sleep loss-driven changes in transcript and protein abundance have been used as a means to better understand the function of sleep for the brain. Here, we use translating ribosome affinity purification (TRAP) to characterize changes in abundance of ribosome-associated transcripts in excitatory and inhibitory neurons in mouse hippocampus and neocortex after a brief period of sleep or sleep loss. We show that these changes are not uniform, but are generally more pronounced in excitatory neurons than inhibitory neurons, and more pronounced in neocortex than in hippocampus.

Received July 12, 2020; revised Mar. 7, 2021; accepted Mar. 24, 2021.

Author contributions: C.P.-M., J.D., and S.J.A. designed research; C.P.-M., J.D., L.W., M.D., and D.P. performed research; S.J. contributed unpublished reagents/analytic tools; C.P.-M., M.D., and D.P. analyzed data; S.J.A. wrote the paper.

This work was supported by National Institutes of Health Research Grants DP2 MH 104119 and R01 NS 104776 and the Human Frontiers Science Program Grant N023241-00_RG105 (to S.J.A.). We thank members of the Aton lab and Dr. Natalie Tronson, Dr. Geoff Murphy, and Dr. Michael Sutton for helpful feedback on this manuscript.

The authors declare no competing financial interests.

Correspondence should be addressed to Sara J. Aton at saton@umich.edu.

https://doi.org/10.1523/JNEUROSCI.1883-20.2021

Copyright $\odot 2021$ Puentes-Mestril et al.

This is an open-access article distributed under the terms of the Creative Commons Attribution 4.0 International license, which permits unrestricted use, distribution and reproduction in any medium provided that the original work is properly attributed.

\section{Introduction}

Sleep is essential for optimal brain function, but the underlying biological mechanisms are largely unknown. Prior work aimed at addressing this question has used molecular profiling of mRNA and protein abundance, in a number of brain areas, to characterize changes caused by experimental sleep deprivation (SD; Cirelli et al., 2004; Mackiewicz et al., 2007; Poirrier et al., 2008; Vecsey et al., 2012; Noya et al., 2019). Transcriptomic changes reported after SD in the brain have led to specific hypotheses regarding the biological underpinnings of cognitive disruptions associated with sleep loss (Dinges et al., 1997; Belenky et al., 2003; Mednick et al., 2003; Stickgold, 2005). For example, the synaptic homeostasis hypothesis (Tononi and Cirelli, 2006) proposes that synapses throughout the brain are 
strengthened during periods of wake and weakened during periods of sleep. The proposal of this hypothesis was initially based on results from transcriptomic studies in mice, showing higher expression of both immediate early genes (IEGs) and several other genes involved in synaptic plasticity after periods of SD versus sleep (Cirelli et al., 1996, 2004; Cirelli and Tononi, 2000; Havekes and Aton, 2020).

However, there may be more heterogeneity in responses to SD across the brain than previously thought. For example, SDdriven transcript changes may vary between different brain structures (Terao et al., 2006; Mackiewicz et al., 2007; Vecsey et al., 2012). We have recently shown that while SD increases expression of the plasticity-mediating IEG Arc and Arc protein abundance in neocortical areas (e.g., primary somatosensory cortex; S1), it simultaneously decreases de novo synthesis of Arc in the hippocampal dentate gyrus (DG). Indeed, recent data have suggested that SD could differentially impact neuronal activity and dendritic spine density in hippocampal versus neocortical structures (McDermott et al., 2003; Vyazovskiy et al., 2009; Havekes et al., 2016; de Vivo et al., 2017; Ognjanovski et al., 2018; Raven et al., 2019; Havekes and Aton, 2020). Because cognitive processes reliant on the hippocampus, such as episodic memory consolidation (Saletin and Walker, 2012; Havekes and Abel, 2017), seem particularly susceptible to disruption by SD, a critical unanswered question is whether SD differentially impacts network activity and plasticity in the two structures. Beyond this, within brain structures, there may be heterogeneity in the responses of different neuronal subtypes to SD. For example, within the neocortex, fast-spiking interneurons, or neurons with greater firing rates, appear to have differential firing rate changes across periods of sleep (Vyazovskiy et al., 2009; Clawson et al., 2018). Consistent with this idea, synaptic excitatory-inhibitory (E-I) balance was recently shown to vary in neocortex over the course of the day in a sleep-dependent manner (Bridi et al., 2020). Moreover, while most neocortical neurons fire at lower rates during slow wave sleep (SWS) versus wake, some subclasses of neocortical neurons are selectively sleep-active (Gerashchenko et al., 2008).

Here we aimed to better characterize brain region-specific and cell type-specific changes evoked in the nervous system during $\mathrm{SD}$. We used cell type-specific translating ribosome affinity purification (TRAP; Sanz et al., 2019) to profile SD-mediated changes in ribosome-associated mRNAs in two prominent hippocampal and neocortical cell types, Camk2a-expressing (Camk2a ${ }^{+}$) pyramidal neurons and parvalbumin-expressing $\left(\mathrm{PV}^{+}\right)$interneurons. Because interactions between these two cell types are critical for mediating state-dependent sensory plasticity and memory consolidation (Aton et al., 2013; Kuhlman et al., 2013; Ognjanovski et al., 2017, 2018), we characterized SD-driven changes in ribosomeassociated transcripts encoding transcription-regulating IEGs, plasticity effector proteins, and circadian clock components in these two cell types. We find that SD generally causes more modest changes to these transcripts in hippocampal versus neocortical circuits, and in $\mathrm{PV}^{+}$interneurons versus Camk2a ${ }^{+}$neurons. Together our data suggest that the effects of SD on the brain are more heterogeneous than previously thought, and indicate regiondependent and cell type-dependent differences in SD's impact which may have important implications for brain function.

\section{Materials and Methods}

Mouse handling and husbandry

All animal procedures were approved by the University of Michigan Institutional Animal Care and Use Committee [PHS Animal Welfare
Assurance number D16-00072 (A3114-01)]. Animals were maintained on a 12/12 h light/dark cycle (lights on at 8 A.M.) with food and water provided ad libitum. Mice expressing Cre recombinase in Camk $2 \mathrm{a}^{+}$ neurons or $\mathrm{PV}^{+}$interneurons (B6.Cg-Tg(Camk2a-cre)T29-1Stl/J or

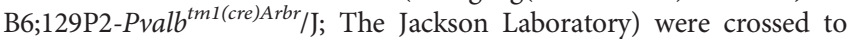
RiboTag mice (B6N.129-Rpl22 $2^{\text {tm1.1Psam }} /$ J; The Jackson Laboratory) to express HA-tagged Rpl22 protein in these neuron populations (Fig. $1 G$ ). Because of the nature of these crosses, this effectively resulted in roughly half of all cellular Rpl22 ribosomal subunit proteins being tagged with HA. Three- to five-month-old male mice were individually housed one week before all experiments (with beneficial enrichment), and were habituated to handling for $5 \mathrm{~d}$ before experiments. Following habituation, and beginning at lights on [zeitgeber time (ZT)0], mice were either allowed ad libitum sleep in their home cage or were SD by gentle handling (Durkin and Aton, 2016; Durkin et al., 2017; Delorme et al., 2019; Clawson et al., 2021). For sleeping animals, sleep behavior was visually scored at 5 min or 2 min intervals (for 6- and 3-h SD, respectively; Figs. $2 B, 3 A$ ), based on immobility and assumption of characteristic sleep postures. Previous research from our lab has shown that wake time over the final $45 \mathrm{~min}$ of the experiment correlates with Arc IEG expression in neocortex (Delorme et al., 2019). Thus, to reduce the probability of confounding results from freely-sleeping mice, mice in the sleep groups that spent $>60 \%$ of the final 45 min of the experiment in wake were excluded from subsequent analysis. All mice were killed with an overdose of pentobarbital (Euthasol) before tissue harvest.

\section{Experimental design and statistical analysis}

For TRAP experiments, male Camk2a::RiboTag and PV::RiboTag mice were randomly assigned to 3 - or 6-h sleep ( $n=4$ and $n=5$, respectively) and SD ( $n=5$ and 6 , respectively) groups. qPCR data were quantified and statistically compared as described below. For each Ribotag-expressing genotype, 6 male mice were used for HA immunohistochemistry as described below. For in situ hybridization, three- to five-month-old male C57Bl/6J mice (The Jackson Laboratory) were randomly assigned to 6-h sleep and SD groups ( $n=6$ each). Image analysis for in situ was conducted as described below.

\section{TRAP}

TRAP was performed using methods described in prior studies (Sanz et al., 2009), with minor modifications. Following 3-6 h of ad libitum sleep or SD, animals were euthanized with an overdose of pentobarbitol (Euthasol), their brains extracted, and hippocampi/cortices dissected in dissection buffer [ $1 \times$ HBSS, 2.5 mм HEPES (pH 7.4), 4 mм $\mathrm{NaHCO}_{3}$, $35 \mathrm{~mm}$ glucose, and $100 \mu \mathrm{g} / \mathrm{ml}$ cycloheximide]. Tissue was then transferred to glass dounce column containing $1 \mathrm{ml}$ of homogenization buffer $[10 \mathrm{~mm}$ HEPES (pH 7.4), $150 \mathrm{~mm} \mathrm{KCl,} 10 \mathrm{~mm} \mathrm{MgCl}_{2}, 2 \mathrm{~mm}$ DTT, cOmplete Protease Inhibitor Cocktail (Sigma-Aldrich, 11836170001), $100 \mathrm{U} / \mathrm{ml}$ Rnasin Ribonuclease Inhibitors (Promega, N2111), and $100 \mu \mathrm{g} / \mathrm{ml}$ cycloheximide] and manually homogenized on ice. Homogenate was transferred to a $1.5-\mathrm{ml}$ LoBind tubes (Eppendorf) and centrifuged at $1000 \times g$ at $4^{\circ} \mathrm{C}$ for $10 \mathrm{~min}$. Supernatant was then transferred to a new tube, $90 \mu \mathrm{l}$ of $10 \% \mathrm{NP}-$ 40 was added, and samples were allowed to incubate for $5 \mathrm{~min}$. Following this step, the supernatant was centrifuged at maximum speed for $10 \mathrm{~min}$ at $4^{\circ} \mathrm{C}$, transferred to a new tube, and mixed with $10 \mu \mathrm{l}$ of HA-antibody (Abcam, ab9110; Jiang et al., 2015; Shigeoka et al., 2018). Antibody binding proceeded by incubating the homogenate-antibody solution for 1.5 $\mathrm{h}$ at $4^{\circ} \mathrm{C}$ with constant rotation. During the antibody rinse, $200 \mu \mathrm{l}$ of Protein G Dynabeads (ThermoFisher, 10009D) were washed three times in $0.15 \mathrm{M} \mathrm{KCl} \mathrm{IP} \mathrm{buffer} \mathrm{[10} \mathrm{mM} \mathrm{HEPES} \mathrm{(pH} \mathrm{7.4),} 150 \mathrm{~mm} \mathrm{KCl,}$ $10 \mathrm{~mm} \mathrm{MgCl}_{2}$, and $1 \% \mathrm{NP}-40$ ] and incubated in supplemented homogenization buffer (10\% NP-40). Following this step, supplemented buffer was removed, the homogenate-antibody solution was added directly to the Dynabeads, and the solution incubated for $1 \mathrm{~h}$ at $4^{\circ} \mathrm{C}$ with constant rotation. After incubation, the RNA-bound beads were washed four times in $900 \mu \mathrm{l}$ of $0.35 \mathrm{M} \mathrm{KCl}[10 \mathrm{~mm}$ HEPES (pH 7.4), 350 mм KCl, 10 mм $\mathrm{MgCl}_{2}, 1 \%$ NP-40, 2 mм DTT, $100 \mathrm{U} / \mathrm{ml}$ Rnasin Ribonuclease Inhibitors (Promega, N2111), and $100 \mu \mathrm{g} / \mathrm{ml}$ cycloheximide]. During the final wash, beads were placed onto the magnet and moved to room temperature. After removing 
the supernatant, RNA was eluted by vortexing the beads vigorously in 350- $\mu$ l RLT (QIAGEN, 79 216). Eluted RNA was purified using RNeasy Micro kit (QIAGEN).

\section{Quantitative real-time PCR ( $q P C R)$ and stability analysis}

qPCR experiments were performed as described previously (Delorme et al., 2019). Briefly, purified mRNA samples were quantified by spectrophotometry (Nanodrop Lite; ThermoFisher) and diluted to equal concentrations. 20-500 ng of mRNA was used to synthesize cDNA using iScript's cDNA Synthesis kit (Bio-Rad), cDNA diluted 1:10 in RNasefree $\mathrm{H}_{2} \mathrm{O}$, and measured using a CFX96 Real-Time System. Primers were designed for these studies, with the exception of Homerla, for which sequences were established in a prior study (Mikhail et al., 2017). Primer specificity was confirmed using NIH Primer Blast (for primer sequences, see Extended Data Table 1-1). Three technical replicates were used for each sample. Relative changes in gene expression between sleep and SD were quantified using the $\Delta \Delta \mathrm{CT}$ method, and these fold changes are presented on a $\log$ scale ( $\log _{2}$ transformed value equivalent to $\Delta \Delta \mathrm{CT}$ ) with propagated errors. All statistical analyses were performed on $\Delta \mathrm{CT}$ values.

Reference (housekeeping) genes for normalization were chosen for each experiment based on three measures: intragroup variability, intergroup variability, and an overall stability measure derived from total variance (Extended Data Tables 1-2, 1-3). Special emphasis was placed on selecting pairs of reference transcripts with countervailing intergroup differences. These measures were calculated using Normfinder (Andersen et al., 2004) and RefFinder (Xie et al., 2012) software. Because Normfinder is better suited for large sample sizes, RefFinder was used to validate Normfinder rankings and ensure genes with low (or opposite-direction) intergroup variability were chosen as housekeeping pairs. Stability measures were calculated for each sleeping condition, region, and mRNA population and repeated for mRNAs purified from $P V::$ RiboTag and Camk2a::Ribotag mice (Table 1). The arithmetic mean of each housekeeping pair was then used to normalize target gene expression. As a final measure of housekeeping stability, we calculated each pairs' fold change between mice in SD and sleep groups.

\section{RNAScope in situ hybridization}

Fluorescent in situ hybridization was performed on $14-\mu \mathrm{m}$ coronal sections taken from fixed-frozen brains of sleep $(n=6)$ and SD $(n=6)$ mice. Section coordinates $(1-3.0 \mathrm{~mm}$ lateral, -1.4 to $-2.8 \mathrm{~mm}$ posterior to bregma) were similarly distributed between sleep and SD conditions (Fig. 3B). The RNAScope Multiplex Fluorescent Reagent kit v2 with 4plex ancillary kit was used to label Arc, Homer1a, Cfos, and Pvalb transcripts (Fig. $3 C-F$ ). Before probe incubation, slices were pretreated with hydrogen peroxide (10 min, room temperature), Target Retrieval reagent $\left(5 \mathrm{~min} 99^{\circ} \mathrm{C}\right)$, and RNscope Protease III $\left(30 \mathrm{~min}, 40^{\circ} \mathrm{C}\right)$. Slices were incubated with custom-synthesized Arc (20 bp, target region: 23-1066, 316911-C3, Advanced Cell Diagnostics), Cfos (20 bp, target region: 4071427, 316921-C1, Advanced Cell Diagnostics), Homerla (6 bp, target region: 1301-1887m 433941-C2, Advanced Cell Diagnostics), and Pvalb 16 (16 bp, target region: 2-885, 421931-C4, Advanced Cell Diagnostics). Probes were chosen so as to overlap with regions amplified by qPCR primer pairs (Extended Data Table 1-1). Arc, Cfos, Homerla, and Pvalb were hybridized to Opal Dyes 620 (FP1495001KT, Akoya Biosciences), 570 (FP1488001KT, Akoya Biosciences), 690 (FP1497001KT, Akoya Biosciences), and 520 (FP1487001, Akoya Biosciences), respectively, for visualization. Positive and negative control probes were used in parallel experiments to confirm the specificity of hybridization signals (Fig. 3G, $H$, Fig. $4 A, B)$.

\section{Immunohistochemistry}

For immunohistochemical validation of appropriately cell targeted HA expression in RiboTag-expressing mice, Camk2a::RiboTag and $P V::$ RiboTag mice from sleep $(n=6)$ and SD $(n=6)$ groups were killed and perfused with PBS followed by $4 \%$ paraformaldehyde; $50-\mu \mathrm{m}$ brain sections were blocked with normal goat serum for $2 \mathrm{~h}$ and incubated
Table 1. Housekeeping pairs used for RiboTag qPCR conditions

\begin{tabular}{llllll}
\hline $\begin{array}{l}\text { mRNA } \\
\text { population }\end{array}$ & \multirow{2}{*}{ Condition } & Region & Gene pair & $\mathrm{SD}\left(2^{-\mathrm{CT}}\right) / \mathrm{S}\left(2^{-\mathrm{CT}}\right)^{1}$ & Fold change $^{2}$ \\
\hline Camk2a & \multirow{2}{*}{$\mathrm{h}$} & Cortex & Actg1/Hprt1 & 0.98 & -1.02 \\
& & Hippocampus & Gapdh/Tuba4a & 0.90 & -1.11 \\
& \multirow{2}{*}{$6 \mathrm{~h}$} & Cortex & Pgk1/Tbp & 0.87 & -1.15 \\
& \multirow{2}{*}{$3 \mathrm{~h}$} & Hippocampus & Gapdh/Tuba4a & 0.92 & -1.08 \\
PV & Cortex & Actg1/Hprt1 & 0.82 & -1.22 \\
& \multirow{2}{*}{$6 \mathrm{~h}$} & Hippocampus & Gapdh/Tuba4a & 1.02 & 1.02 \\
& & Cortex & Pgk1/Tbp & 0.97 & -1.03 \\
& & Hippocampus & Gapdh/Tuba4a & 1.02 & 1.02 \\
\hline
\end{tabular}

Change in gene expression presented as ratio ${ }^{1}$ and fold change ${ }^{2}$.

overnight using biotin-conjugated anti-HA (Biolegend 901505, 1:500) and anti-PV (Synaptic Systems 195004, 1:500) antibodies at $4^{\circ} \mathrm{C}$. The following day, sections were stained with streptavidin-Alexa Fluor 647 (Biolegend 405237) and Alexa Fluor 555 goat anti-guinea pig IgG H\&L (Abcam ab150186). Stained sections were coverslipped in ProLong Gold Antifade Reagent (ThermoFisher, P36930). Fluorescence intensity was used to identify HA-expressing $\left(\mathrm{HA}^{+}\right)$cells, $\mathrm{PV}^{+}$cells, and overlapping cells within the DG, CA1, CA3, and neocortex. To account for differences in localization and spread of antibody staining, both $\mathrm{PV}^{+} \mathrm{HA}^{+}$cells and $\mathrm{HA}^{+} \mathrm{PV}$-expressing cells were identified, and overlap was quantified in terms of both cell count and cell area (Fig. $1 A-F)$. Quantification was performed using the semi-automated protocol detailed below. Camk2a antibody staining was not used, as widespread diffuse neuropil labeling made accurate cell counting (i.e., detection of immunonegative cell bodies) infeasible.

\section{Imaging and quantification}

RNAScope probe fluorescence signals were captured and stitched using a $40 \times$ objective lens on a Leica 3D STED SP8. Immunostained brain sections were imaged on a Leica SP5 laser scanning confocal microscope. Settings were fixed for each imaging session. Fluorescence images were analyzed using MIPAR image analysis software in their raw grayscale format (Sosa et al., 2014). Two images per region (one per hemisphere) were quantified for each animal. Quantification was performed separately for regions CA1, CA3, and DG in dorsal hippocampus, and layers $2 / 3,4,5$, and 6 of overlying (i.e., dorsal) neocortical regions (including S1). Total fluorescence dot number and average intensity of a single dot calculated per the recommended guidelines provided by ACDBio (ACDBio, 2017), for Pvalb+ and non-Pvalb+ regions of interest (ROIs) within granule (DG), pyramidal (CA1, CA3), and cortical layers 1-6 (layers were manually isolated using a freehand tool by a scorer blind to experimental condition). Fluorescence intensity and expression overlap were calculated using a semi-automated protocol curated by blinded scorer. Briefly, a non-local means filter was used to reduce image noise, and an adaptive threshold was used to identify areas $>30 \mu \mathrm{m}$ whose mean pixel intensity was $200 \%$ of its surroundings. Identified areas were labeled as IEG + or Pvalb + and manually edited to refine labeling, select for representative dots, and remove artifacts (manual editing was not used to label any additional IEG expression). Finalized labeling was used to delineate $P v a l b+$ and non-Pvalb + ROIs, select for background area (area in the ROI minus areas of labeled expression), and identify IEG+ $P v a l b+$ cells (Fig. 5). Intensity values from ROIs, background, and selected dots were used to calculate fluorescence dots/area. Average background intensity was calculated as the fluorescence intensity of the selected background area per unit area. The average intensity of a single fluorescent dot was calculated for each transcript as the intensity of manually selected representative dots within the ROI, minus the average background intensity multiplied by the area, divided by the total number of selected dots. Dot intensity values did not differ between sleep and SD mice for specific transcripts. The total fluorescent dot number within each ROI was calculated by subtracting average background intensity from total ROI fluorescence intensity, multiplied by total area, divided by average dot intensity. 

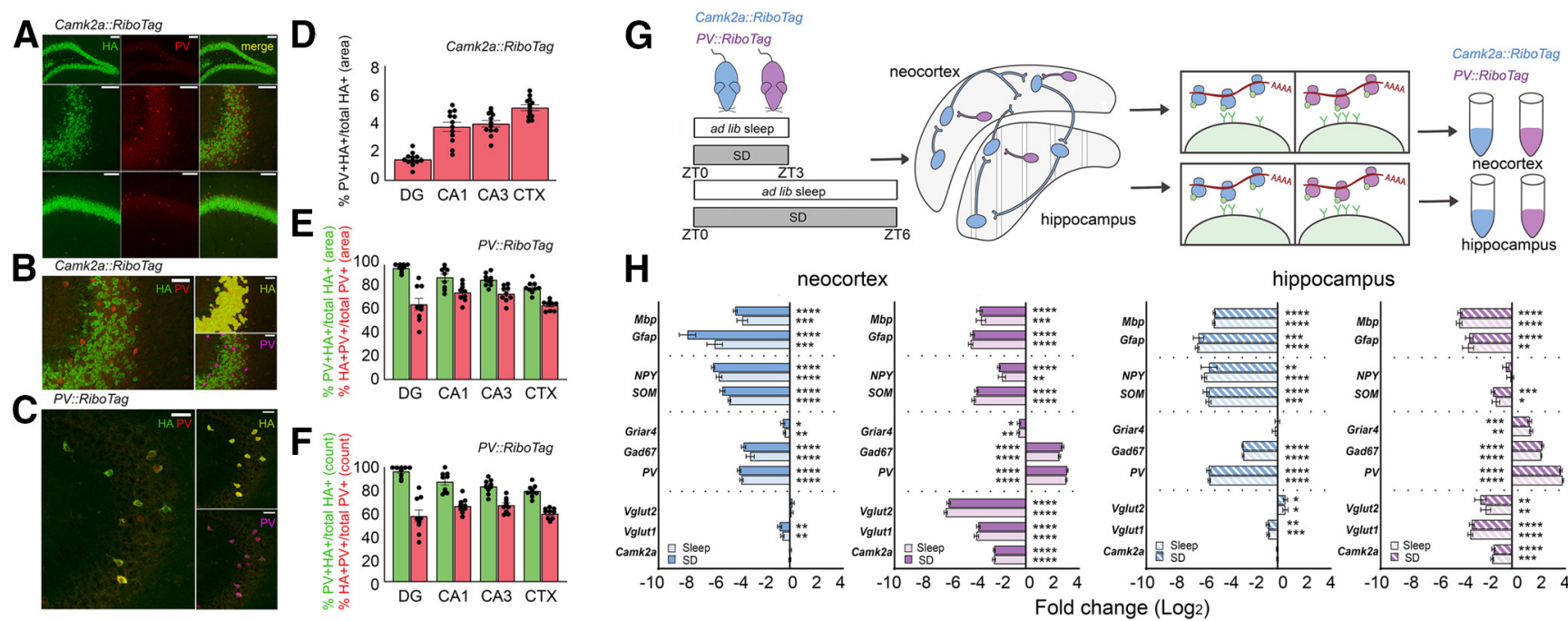

Figure 1. Validation and experimental design for TRAP. A, Antibody staining for Rpl22-HA (green) and PV (red) is shown for dorsal hippocampal subregions DG (top), CA3 (middle), and CA1 (bottom) of a Camk2a::RiboTag mouse. Scale bars: $100 \mu \mathrm{m}$. B, Example of automated protocol used for Camk2a::RiboTag mice to quantify non-specific expression (i.e., overlap of PV ${ }^{+}$with $\mathrm{HA}^{+}$expression regions; quantified in $\boldsymbol{D}$ within the pyramidal cell layer of CA3). Areas of overlapping fluorescence were determined using automated detection of $\mathrm{HA}^{+}$(green fluorescence, labeled in yellow) and $\mathrm{PV}^{+}$(red fluorescence, labeled in magenta) areas. Scale bars: $50 \mu \mathrm{m}$. C, HA expression in $\mathrm{PV}^{+}$interneurons was validated with immunohistochemistry in PV::RiboTag mice, using the method described in $\boldsymbol{B}$. D, Areas of $\mathrm{PV}^{+}$and $\mathrm{HA}^{+}$overlap (as a proportion of total $\mathrm{HA}^{+}$area) in Camk2a::RiboTag sections were minimal. $\boldsymbol{E}, \boldsymbol{F}, \mathrm{HA}^{+}$and $\mathrm{PV}^{+}$overlapping expression presented as a proportion of total $\mathrm{PV}^{+}$or $\mathrm{HA}^{+}$cell counts (red and green, respectively; $\boldsymbol{E}$ ) and total $\mathrm{PV}^{+}$or $\mathrm{HA}^{+}$area (red and green, respectively, $\boldsymbol{F}$ ) in $P V:: R i b o T a g$ sections. $\boldsymbol{G}$, Experimental design for cell type-specific ribosomal profiling. Camk2a::RiboTag (blue) and PV::RiboTag (violet) transgenic mice were killed after a 3- or 6-h period of ad libitum sleep (sleep) or SD starting at lights on (ZTO). Ribosome-associated mRNAs were affinity purified from hippocampus and neocortex separately. $\boldsymbol{H}$, Enrichment of markers for glia (Mbp, Gfap), non-PV ${ }^{+}$inhibitory neurons (NPY, SOM), PV ${ }^{+}$neurons (Griar4, Gad67, Pvalb), and excitatory neurons (Vglut1, Vglut2, Camk2a) calculated as $\Delta \Delta$ CT between affinity purified (RiboTag) mRNA and input mRNA from neocortex or hippocampus. Data presented as $\log (2)$ transformed fold changes and are shown separately for SD and sleep conditions. Gene expression was normalized to housekeeping gene pairs according to their respective condition (see Table 1). Values indicate mean \pm SEM with propagated error; ${ }^{*} p<0.05$, ${ }^{* *} p<0.01$, ${ }^{* * *} p<0.001$, ${ }^{* * * *} p<0.0001$, one sample $t$ test against a hypothetical value of 0 .

\section{Results}

TRAP-based characterization of ribosome-associated transcripts in Camk2 $\mathrm{a}^{+}$and $\mathrm{PV}^{+}$neuronal populations To quantify how ribosome-associated transcripts in different neuronal populations within the hippocampus and neocortex are affected by sleep loss, we crossed RiboTag transgenic mice (with Cre recombinase-dependent expression of HA-tagged Rpl22 protein) to either Camk2a-Cre or PV-Cre transgenic lines (Sanz et al., 2019). Appropriate cell type-specific expression of $\mathrm{Rpl} 22^{\mathrm{HA}}$ in Camk2a::RiboTag and PV::RiboTag mice was verified using immunohistochemistry (Fig. $1 A-C$ ). HA expression was largely circumscribed to the intended cell type. For example, expression of HA in Camk2a::RiboTag was appropriately localized to cell bodies in the pyramidal cell layer of hippocampal areas CA1, CA3, and DG (Fig. 1A). Expression of HA in non-targeted cell types of the hippocampus (e.g., colabeling for $\mathrm{PV}$ in $\mathrm{HA}^{+}$cells in Camk2a::RiboTag mice) was minimal (3.6 $\pm 0.2 \%$ on average; Fig. 1D). In the hippocampus and neocortex of $P V::$ RiboTag mice, $86.7 \pm 1.5 \%$ and $79.4 \pm 1.8 \%$ of $\mathrm{HA}^{+}$neurons, respectively, co-expressed PV peptide; similar values for overlap were calculated when either the total area of HA-immunopositive and $\mathrm{PV}$-immunopositive regions within these structures, or immunopositive cell counts, were directly compared (Fig. 1E,F). We next validated cell type-specificity of ribosome-associated transcripts isolated from transgenic mouse lines. Following a period of ad libitum sleep of SD starting at lights on (ZT0), hippocampi and neocortex were dissected, and ribosome-associated mRNAs were isolated (Sanz et al., 2019). We compared abundance of cell type-specific transcripts between RiboTag affinity purified mRNA and Input mRNA from whole hippocampus or neocortex homogenate using qPCR (Fig. 1G). Enrichment or de-enrichment of these cell markers was compared with a null hypothetical value of 0 using one-sample $t$ tests. We found that ribosomal- associated transcripts from both the neocortex and hippocampus of Camk2a::RiboTag mice de-enriched for markers of glial cell types $(M b p, G f a p)$, non-PV ${ }^{+}$inhibitory neurons (Npy, Sst), PV interneurons (Gad67, Pvalb), and Vglut1 relative to input (Fig. $1 H$ ). Hippocampal enrichment patterns mirrored those of the neocortex with the exception of Vglut2, which was significantly enriched relative to Input. Ribosome-associated transcripts from $P V:: R i b o T a g$ mice de-enriched for markers of glial (Mbp, Gfap), non- $\mathrm{PV}^{+}$inhibitory (Npy, Sst), and excitatory neurons (Vglut1, Vglut2, Camk2a) while enriching for $\mathrm{PV}^{+}$interneuron markers (Pvalb, Gad67) relative to Input. We made comparisons of cell type-specific transcript enrichment separately for mice which were either allowed ad libitum sleep or SD over the first 3 or $6 \mathrm{~h}$ after lights on (i.e., from ZT0 to ZT3 or ZT0 to ZT6). No substantial differences in enrichment patterns were observed between sleep and SD mice (not significant (N.S.), Holm-Sidak post hoc test). These data confirm the high degree of specificity of TRAP-based profiling for ribosomal transcripts from Camk $2 \mathrm{a}^{+}$ principal neurons and $\mathrm{PV}^{+}$interneurons.

SD-driven changes in ribosome-associated plasticity-related mRNAs and activity-dependent transcription regulators vary with cell type and brain structure

We first quantified a subset of transcripts encoding for proteins involved in synaptic plasticity (i.e., plasticity effectors) whose expression levels have been reported previously as altered by SD, Arc, Homer1a, Narp, and Bdnf (Cirelli et al., 2004; Maret et al., 2007). Ribosome-associated transcript abundance was first quantified in Camk $2 \mathrm{a}^{+}$neocortical and hippocampal neuron populations after $3 \mathrm{~h}$ of ad libitum sleep (sleep; $n=4)$ or $\mathrm{SD}(n=5)$, starting at lights on (ZT0; Fig. $2 A-C$ ). Consistent with previous findings (Cirelli et al., 2004), 3-h SD significantly increased neocortical Arc $(p<0.001$, Holm-Sidak post hoc test $)$ and Homerla 
A

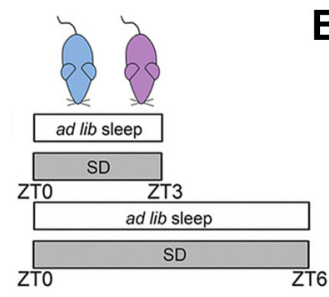

B

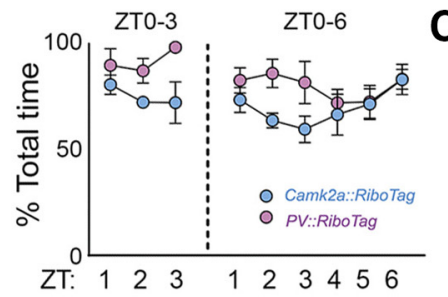

C

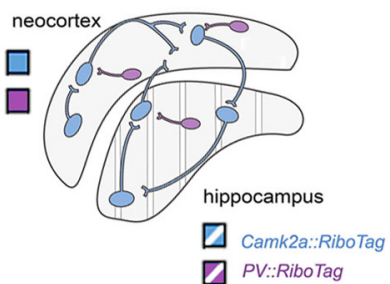

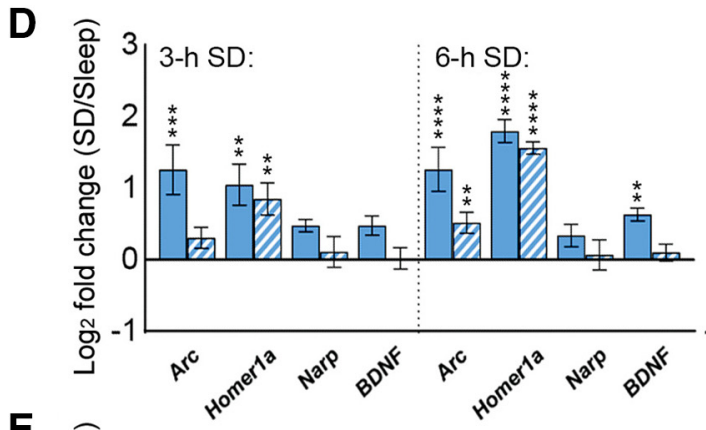

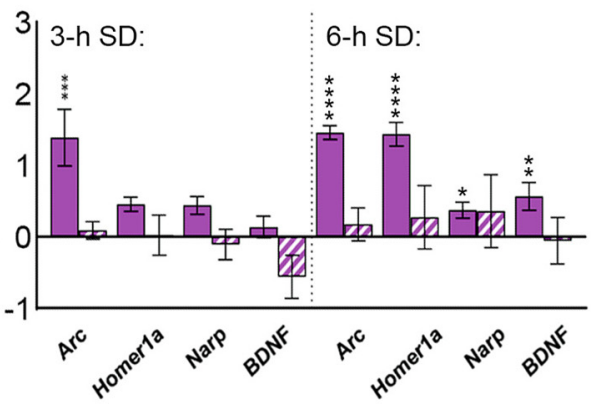

E

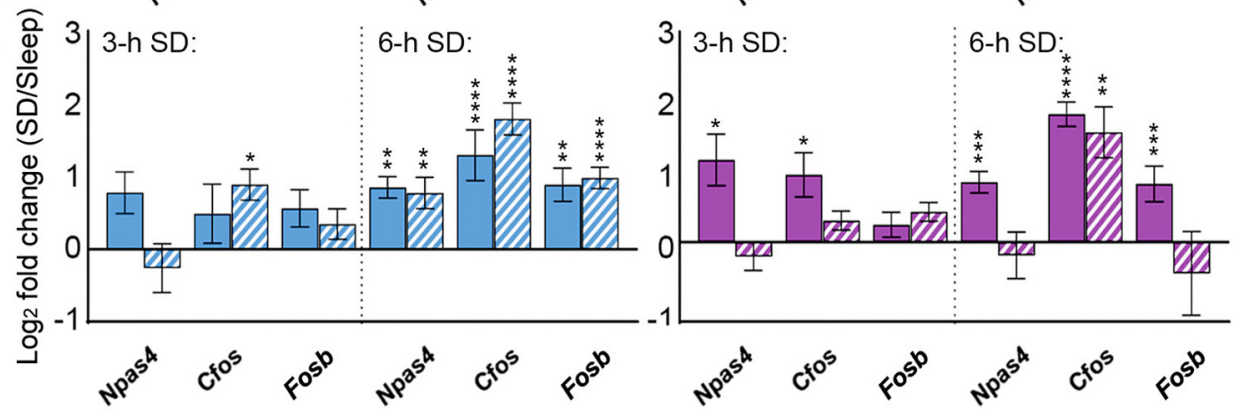

Figure 2. SD increases ribosome-associated plasticity effector transcripts and immediate-early transcription regulators in a cell type-specific and region-specific manner. $\boldsymbol{A}$, Experimental design for sleep versus SD. B, Sleep amounts for 3- and 6-h ad libitum sleep groups, calculated as a percent of total time. C, SD effects on ribosome-associated transcript abundance were quantified separately from neocortex and hippocampus from Camk2a::RiboTag (blue) and PV::RiboTag (violet) transgenic mice. D, Three-hour SD significantly increased Arc and Homer1a levels on ribosomes from Camk2a ${ }^{+}$neocortical (solid) neurons; only Homer1a increased in hippocampal (dashed) neurons. Three-hour SD significantly increased Arc on ribosomes from PV ${ }^{+}$interneurons in neocortex; no significant change was observed in the hippocampal PV ${ }^{+}$interneuron population. Arc, Homer1a, and Bdnf significantly increased after 6-h SD in Camk2a ${ }^{+}$neocortical neurons; Arc and Homer1a were increased within the Camk2a ${ }^{+}$hippocampal population. All effector transcript levels were significantly elevated after 6-h SD in PV ${ }^{+}$interneurons in neocortex; no significant change was observed in the hippocampal PV ${ }^{+}$population. Transcript level changes are presented as a $\log _{2}$ fold change between SD and ad libitum sleep mice. E, Three-hour SD had no significant effect on IEG transcript levels on ribosomes from Camk2a ${ }^{+}$neocortical (solid) neurons; only (fos increased in hippocampal (dashed) neurons. Three-hour SD significantly increased Npas4 and Cfos on ribosomes from $\mathrm{PV}^{+}$interneurons in neocortex, but did not affect IEG abundance on ribosomes from hippocampal PV ${ }^{+}$neurons. Six-hour SD significantly increased Npas4, Cfos, and Fosb levels in Camk2a ${ }^{+}$neocortical neurons, Camk2a ${ }^{+}$hippocampal neurons, and $\mathrm{PV}^{+}$neocortical interneurons. Only Cfos significantly increased in the $\mathrm{PV}^{+}$hippocampal population with 6-h SD. Values indicate mean \pm SEM with propagated error; ${ }^{*} p<0.05,{ }^{* *} p<0.01$, ${ }^{* * *} p<0.001$, ${ }^{* * * *} p<0.0001$, Holm-Sidak post hoc test versus sleep.

$(p<0.01$; Maret et al., 2007) ribosome-associated mRNA (Fig. $2 D$; Table 2). In contrast, 3-h SD significantly increased Homerla abundance on hippocampal ribosomes $(p<0.01)$, but did not significantly affect Arc abundance (N.S., Holm-Sidak post hoc test). This is consistent with recent data showing that brief SD results in either no change, or a decrease, in Arc expression in various hippocampal subregions (Delorme et al., 2019). Overall patterns of transcript abundance for the plasticity-regulating proteins $B d n f$ and Narp followed a similar trend, with unchanged levels in hippocampal Camk $2 \mathrm{a}^{+}$neurons (N.S, Holm-Sidak post hoc test), and modestly (but not significantly) increased levels in neocortical neurons (Narp and Bdnf, N.S.). After more prolonged ( $6 \mathrm{~h}) \mathrm{SD}$ ( $n=6 \mathrm{mice} /$ group), ribosomeassociated Arc $(p<0.0001)$, Homerla $(p<0.0001)$, and Bdnf $(p<0.01)$ transcripts were all increased in neocortical Camk $2 \mathrm{a}^{+}$ neurons, whereas Arc $(p<0.01)$ and Homerla $(p<0.0001)$ were increased in hippocampal Camk $2 \mathrm{a}^{+}$neurons (Fig. 2D; Table 2).

We next quantified ribosome-associated transcript abundance in $\mathrm{PV}^{+}$interneuron populations from the neocortex $(n=4$ mice/group) and hippocampus ( $n=4$ and $n=5$ mice for sleep and SD; Table 3). Three-hour SD significantly increased Arc
( $p<0.001$, Holm-Sidak post hoc test) abundance in neocortical $\mathrm{PV}^{+}$interneurons, but had no effect on transcript abundance for plasticity-related proteins in hippocampal $\mathrm{PV}^{+}$interneurons (N.S., Holm-Sidak post hoc test). Six-hour SD increased abundance of these transcripts in the neocortical $\mathrm{PV}^{+}$interneuron population ( $n=5$ and $n=6$ mice for sleep and SD) in a manner similar to the Camk2 $\mathrm{a}^{+}$neuronal population (Arc, $p<0.0001$; Homer1a, $p<0.0001$; Narp, $p<0.05$; Bdnf, $p<0.01)$. In contrast, 6-h SD caused no significant change in any of the ribosome-associated transcripts' abundance in hippocampal $\mathrm{PV}^{+}$ interneurons ( $n=6$ mice/group).

To better characterize how SD affects activity-regulated pathways in Camk2a ${ }^{+}$and $\mathrm{PV}^{+}$populations, we quantified ribosome-associated transcript abundance for IEGs encoding transcription regulatory factors, Npas4, Cfos, and Fosb. We first quantified transcript abundance in Camk $2 \mathrm{a}^{+}$neocortical and hippocampal neuronal populations after $3 \mathrm{~h}$ of ad libitum sleep (sleep; $n=4)$ or SD $(n=5)$, starting at lights on (ZT0). Threehour SD produced no significant change in ribosome-associated transcript abundance in Camk2a ${ }^{+}$neocortical cells (N.S. for all transcripts, Holm-Sidak post hoc test) while significantly 
Table 2. SD-Induced changes in ribosome-associated transcript abundance in Camk2a::RiboTag mice

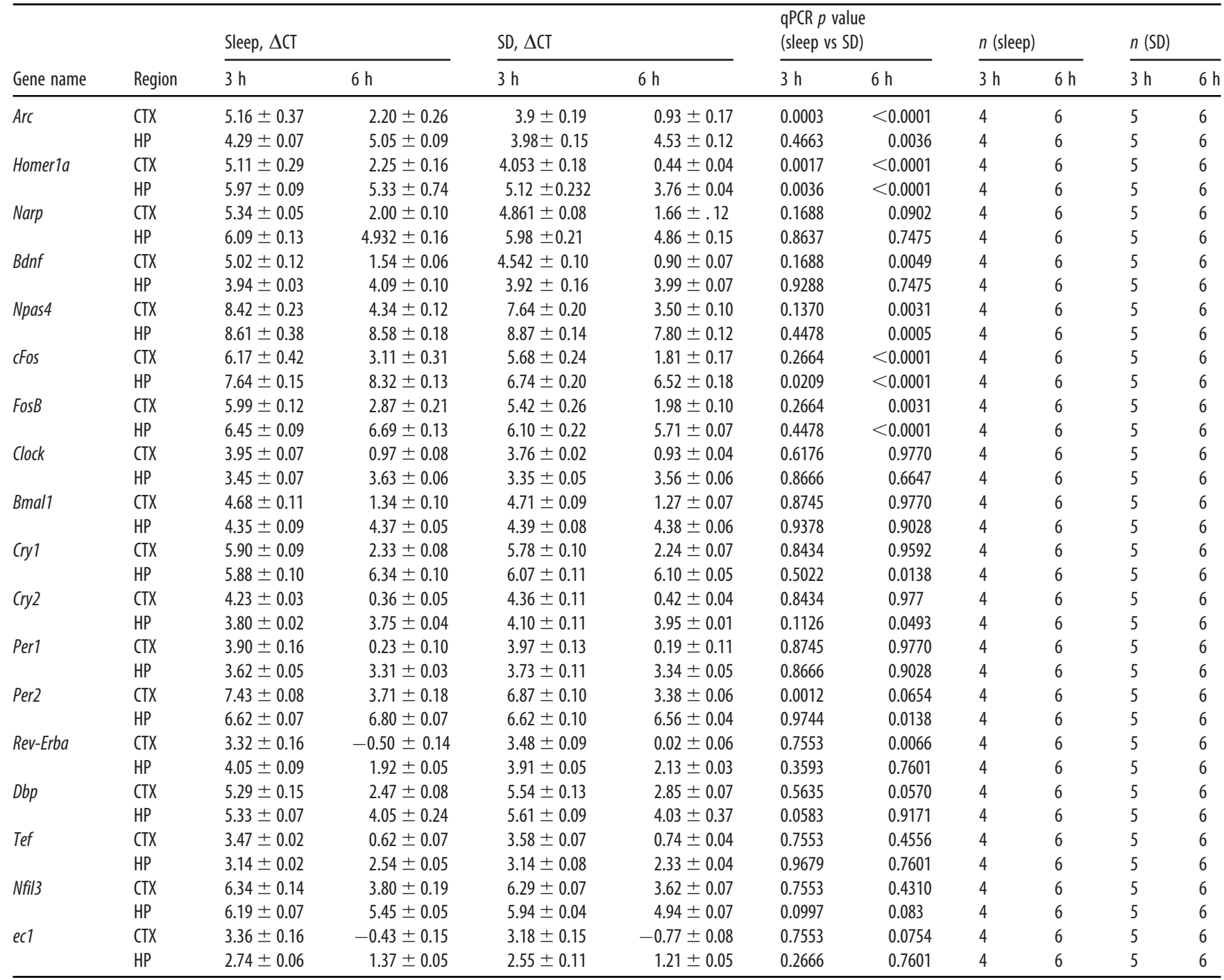

increasing Cfos abundance in the hippocampus ( $p<0.05$; Fig. $2 E$; Table 2). After prolonged (6 h) SD, neocortical Npas4 $(p<0.01)$, $C f o s(p<0.0001)$ and Fosb $(p<0.01)$ abundance increased on ribosomes in Camk $2 \mathrm{a}^{+}$neurons. In the hippocampus, ribosome-associated Npas4 $(p<0.001)$, Cfos $(p<0.0001)$, and Fosb $(p<0.0001)$ all increased in abundance in Camk $2 \mathrm{a}^{+}$neurons after 6-h SD.

We next quantified ribosome-associated transcripts encoding IEG transcription factors in $\mathrm{PV}^{+}$interneurons from the neocortex ( $n=4$ mice/group) and hippocampus ( $n=4$ and $n=5$ mice for sleep and SD; Table 3). Three-hour SD significantly increased neocortical Npas4 and Cfos $(p<0.05)$ abundance, but had no effect on transcript abundance in the hippocampus (N.S. for all transcripts, Holm-Sidak post hoc test). Six-hour SD significantly increased all three transcripts' abundance ( $p<0.0001$ for Cfos, $p<0.001$ for all other transcripts) in the neocortex, but only affected Cfos in the hippocampus $(p<0.01)$. Overall, ribosome-associated transcript abundance in $\mathrm{PV}^{+}$interneurons from the neocortex underwent fold changes that were slightly higher than hippocampus.

Subregion-specific and layer-specific effects of SD on mRNA abundance in pvalb + and non-Pvalb + neurons

Recent findings suggest that effects of SD on transcription and translation may be more region-specific and subregion-specific than previously thought (Delorme et al., 2019; Havekes and Aton, 2020). To more precisely characterize region-specific and cell type-specific changes in overall mRNA abundance after SD, and build on results from TRAP experiments, we used fluorescence in situ hybridization to visualize Pvalb, Arc, Homer1a, and Cfos transcripts in C57B16/J mice after 6-h SD $(n=6)$ or ad libitum sleep ( $n=5$; Figs. $3 A-H, 4 A, B)$. Transcripts were quantified separately in neocortical layers 1-6 and dorsal hippocampal areas DG, CA3, and CA1. Pvalb expression was used to discriminate expression in $\mathrm{PV}^{+}$interneurons from that in non- $\mathrm{PV}^{+}$(mainly pyramidal) neurons. ROIs for Pvalb+ interneurons and non$P v a l b+$ regions were identified separately and total transcript expression (total fluorescence dot number) was calculated relative to background signal and normalized to the area of their respective ROI. We first quantified mRNA abundance after sleep versus $\mathrm{SD}$ among non-Pvalb + cells in neocortical regions overlying dorsal hippocampus (including S1; Fig. 3C). Across neocortex as a whole, SD significantly increased $A r c$ in non-Pvalb+ neurons $\quad\left(\right.$ sleep $=24.8 \pm 10.3$ vs $\mathrm{SD}=79.2 \pm 10.1 \mathrm{dots} / \mathrm{mm}^{2}$, $p<0.05$, Holm-Sidak post hoc test), and showed a tendency for increasing Cfos (sleep $=8.6 \pm 3.9$ vs $\mathrm{SD}=26.2 \pm 5.1 \mathrm{dots} / \mathrm{mm}^{2}$, $p=0.053$ ) and Homerla (sleep $=1.4 \pm 0.5$ vs $\mathrm{SD}=7.8 \pm 2.6$ dots/ $\left.\mathrm{mm}^{2}, p=0.056\right)$. Expression was also quantified in individual neocortical layers. The largest effects of SD were seen for 
Table 3. SD-induced changes in ribosome-associated transcript abundance in PV::RiboTag mice

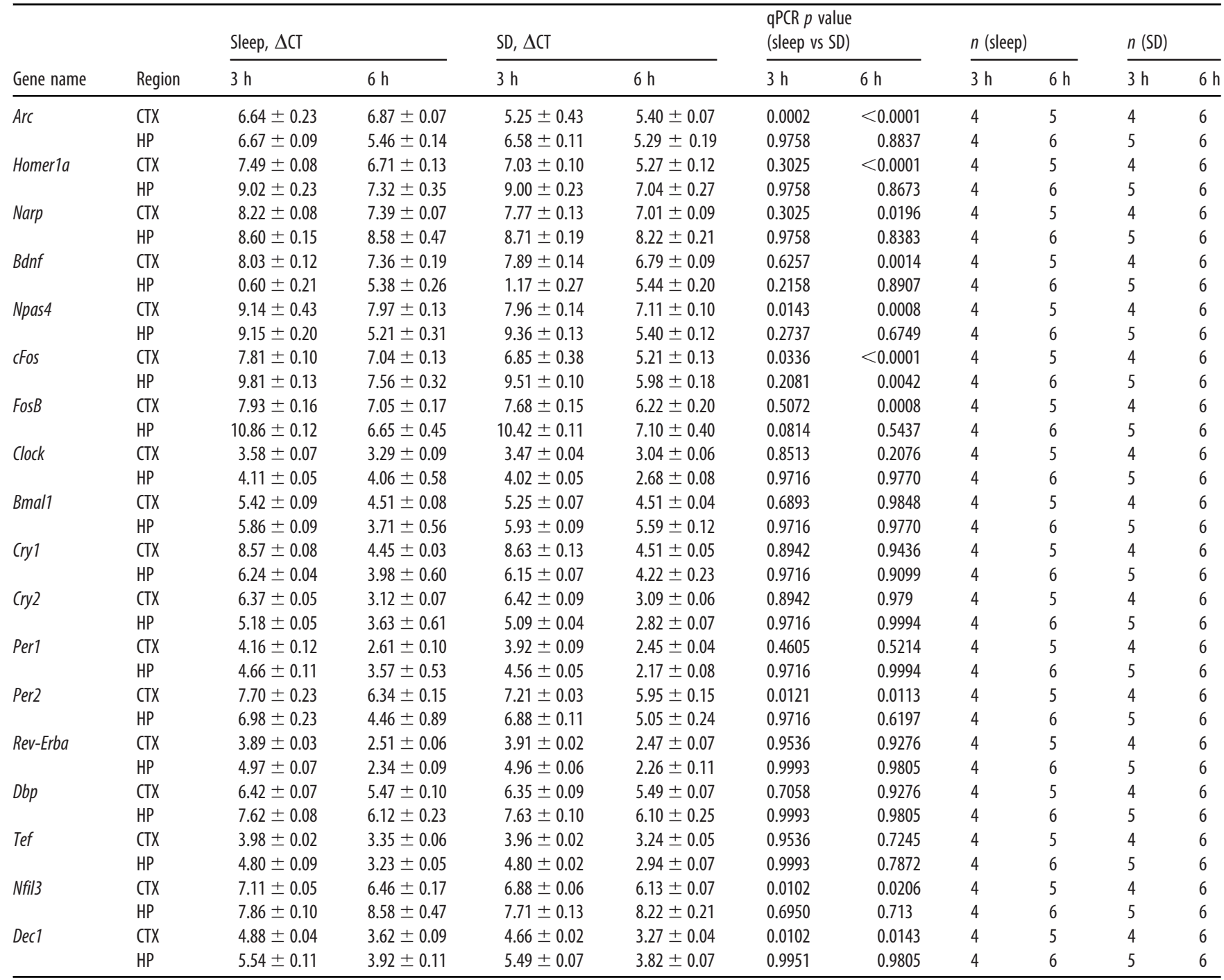

Homer1a and Cfos in layers 4 (Homer1a: sleep $=1.6 \pm 0.6$ vs $\mathrm{SD}=7.8 \pm 2.2$ dots $/ \mathrm{mm}^{2}$, Cfos: sleep $=13.5 \pm 6.4$ vs $\mathrm{SD}=40.5 \pm$ 7.1 dots $/ \mathrm{mm}^{2}$ ) and 5 (Homerla: sleep $=1.5 \pm 0.4$ vs SD $=9.52 .8$ dots $/ \mathrm{mm}^{2}$, Cfos: sleep $=8.8 \pm 3.8$ vs $\mathrm{SD}=34.5 \pm 6.9$ dots $/ \mathrm{mm}^{2}$, $p<0.05)$. SD increased $A r c$ dots $/ \mathrm{mm}^{2}$ significantly across layers $2 / 3 \quad\left(\right.$ sleep $=15.2 \pm 5.8$ vs $\mathrm{SD}=45.8 \pm 3.7 \mathrm{dots} / \mathrm{mm}^{2}, p<0.01$, unpaired $t$ test), 4 (sleep $=36.3 \pm 14.3$ vs $S D=137.5 \pm 17.7$ dots/ $\mathrm{mm}^{2}, p<0.01$ ), and 5 (sleep $=21.7 \pm 8.2$ vs $\mathrm{SD}=81.7 \pm 12.8$ dots $/ \mathrm{mm}^{2}, p<0.05$; Fig. $3 I$ ). No changes in expression were observed with SD in layer 6, and layer 1 expression was not analyzed because of low overall expression and cell density.

In dramatic contrast to the relatively large changes in IEG transcript abundance in putative pyramidal cells in neocortex following SD, neither Arc nor Homer1a (N.S., Holm-Sidak post hoc test) levels were significantly altered by SD in non-Pvalb + cells in any region of dorsal hippocampus (Fig. 4C). Cfos was increased significantly in non-Pvalb+ cells with $\mathrm{SD}$ in CA3 only (sleep $=2.8 \pm 0.5$ vs $\mathrm{SD}=10.7 \pm 1.4$ dots $\left./ \mathrm{mm}^{2}, p<0.01\right)$ with no significant changes in CA1 or DG (N.S., Student's $t$ test). This differs from findings using TRAP (Fig. $2 D, E$ ), where all three transcripts were increased on translating ribosomes from both the neocortex and hippocampus of Camk2a::RiboTag mice after 6-h SD.

We then quantified transcript abundance within $\mathrm{PV}^{+}$interneurons, using Pvalb mRNA expression to define the Pvalb+
ROI (Fig. 3C-F). Overall, IEG expression in Pvalb + cells was relatively low. SD caused no significant changes in Arc or Homerla in any layer of the neocortex, although $C f o s$ dots $/ \mu \mathrm{m}^{2}$ increased selectively in Pvalb + cells in layer $2 / 3$ (sleep $=0.014 \pm 0.002$ vs $\mathrm{SD}=0.043 \pm 0.009 \mathrm{dots} / \mu^{2}, p<0.01$; Fig. $\left.3 J\right)$. Because many Pvalb + cells expressed no detectable IEGs, we also quantified expression within the subpopulation of $P v a l b+$ interneurons which had detectable levels of mRNA expression. Using a semiautomated protocol for this more circumscribed analysis, we found that SD did not affect expression levels for Arc or Cfos, but did increase Homerla dots/ $\mu \mathrm{m}^{2}$ when measured across the entire neocortex (Fig. $3 K$ ). Thus, in contrast to significant increases in ribosome-associated transcripts in neocortical $\mathrm{PV}^{+}$interneurons observed after 6-h SD (Fig. 2D,E), changes in total transcript levels in these cells (measured with in situ hybridization) were relatively minimal. Consistent with the generally limited ribosomeassociated transcript changes observed in hippocampus with SD (Fig. 2D,E), no significant changes in IEG expression were observed in Pvalb+ interneurons from any region of dorsal hippocampus with SD, regardless of method for quantification (Fig. $4 D, E)$.

One possibility is that the relative proportion of $\mathrm{IEG}^{+} \mathrm{PV}^{+}$ interneurons varied as a function of SD. Because Pvalb+ interneurons varied substantially in terms of ROI size, we quantified 
A
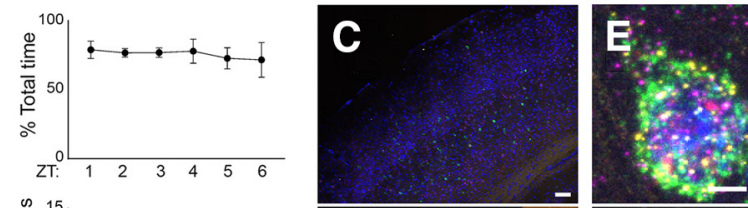

B
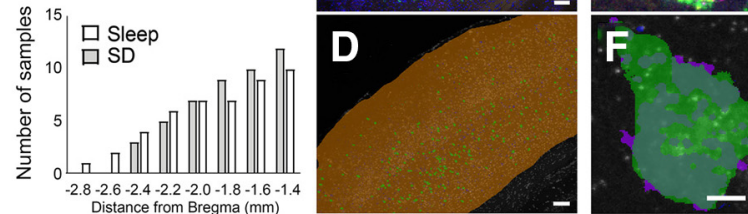

G
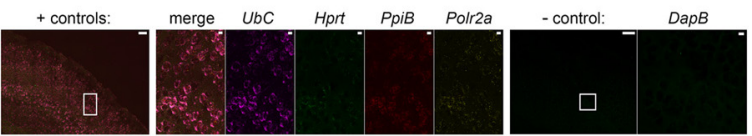

H

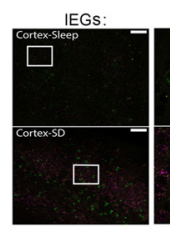

Arc

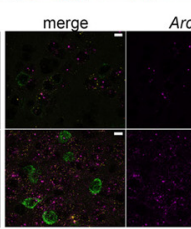

I

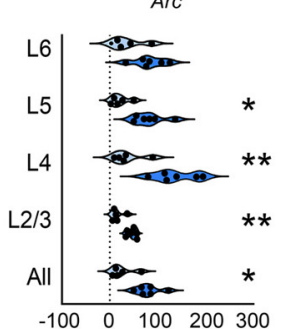

J

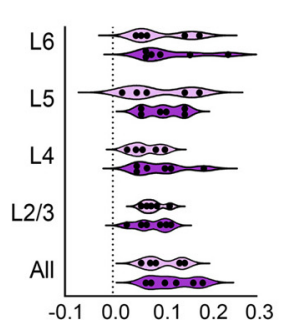

K
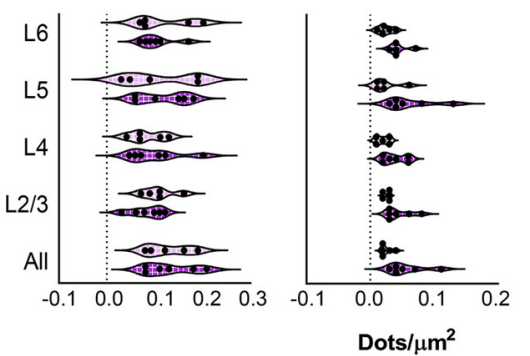

Figure 3. Layer-specific and cell type-specific induction of IEG expression in neocortex after SD. A, Proportion of time spent in ad libitum sleep between ZT0 and ZT6 for mice used for fluorescence in situ hybridization experiments. $B$, Cumulative frequency distribution of $A$ / P coordinates (relative to bregma) for brain sections used in analysis. C, A representative image of neocortical in situ hybridization for Arc (magenta), Cfos (yellow), Homer1a (red), and Pvalb (green). DAPI staining shown in blue. $\boldsymbol{D}$, Anatomical regions for quantification were demarcated manually (shown in orange). Within these anatomic regions, Pvalb (green) fluorescence delineated Pvalb + and non- Pvalb + ROls. An automated protocol was used to calculate the total fluorescence intensity and area of each ROI (and background). Scale bars: $100 \mu \mathrm{m}$. E, Example of IEG and Pvalb fluorescence. $\boldsymbol{F}$, Pvalb + ROl demarcation. Scale bar: $5 \mu \mathrm{m}$. $\boldsymbol{G}$, Representative images showing neocortical riboprobe labeling for ubiquitously expressed (+ control) housekeeping genes UbC (magenta), Hprt1 (green), PpiB (red), and Polrza (yellow). Negative control probes targeting DapB mRNA, a gene expressed in Bacillus the IEG + proportion of Pvalb + interneurons in sleep and SD mice, as a function of both cell count and ROI area (Fig. 5). We found that SD significantly increased the proportion of $\mathrm{Arc}+$ and $C f o s+P v a l b+$ interneurons in the neocortex, across all layers quantified (Fig. 5B). No significant differences were observed in the proportion of Homerla + Pvalb + interneurons. Similarly, we found significant increases in the proportion of $A r c+$ and $C f o s+P v a l b+$ area after SD for all neocortical layers, with the exception of layer 5 . No differences were observed for Homerla + area with $\mathrm{PV}^{+}$interneurons using this measure. No significant changes in any of the mRNAs' expression were observed after SD in Pvalb+ interneurons in any region of the hippocampus after SD, regardless of the method of quantification (Fig. 5C).

Critically, Pvalb expression itself can be regulated as a function of synaptic plasticity (Donato et al., 2013). We found that when expression values were calculated cell by cell, Pvalb levels did vary in both DG and neocortex as a function of SD (values plotted as cumulative distributions in Fig. 5D). These changes moved in opposite directions, with DG neurons showing SDdriven decreases in Pvalb labeling intensity, and neocortex showing SD-driven increases in Pvalb.

Together, our in situ hybridization data suggest that SD: (1) drives relatively modest changes in Homerla, Arc, and Cfos in neocortical $\mathrm{PV}^{+}$interneurons; (2) does not affect these transcripts in hippocampal $\mathrm{PV}^{+}$interneurons; and (3) drives differential changes in expression of Pvalb expression in hippocampal versus neocortical $\mathrm{PV}^{+}$interneurons.

\section{Cell type-specific and region-specific effects of SD on ribosome-associated transcripts involved in circadian timekeeping}

SD has previously been implicated in regulating core molecular clock genes' expression (Bolsius et al., 2021). This effect of SD may be similar to regulation of IEG expression, particularly as clock genes such as Per 1 and Per 2 can be regulated by cellular activity in a manner similar to other IEGs (Balsalobre et al., 1998; Kuhlman et al., 2003; Lee et al., 2010). However, similar to other IEGs, the extent to which SD differentially impacts core clock gene expression as a function of cell type and brain region is unclear. Consequently, we quantified ribosome-associated transcript abundance for core clock genes, Clock, Per1, Per2, Cry1, Cry1, and Bmal1, after SD in Camk2a ${ }^{+}$neurons and $\mathrm{PV}^{+}$interneurons of the neocortex and hippocampus (Fig. 6A). Consistent with findings from whole neocortical tissue (Franken et al., 2007; Hoekstra et al., 2019), we found that 3-h SD significantly increased Per 2 expression in neocortical Camk $2 \mathrm{a}^{+}$neurons and $\mathrm{PV}^{+}$interneurons (Fig. 6B). In contrast, SD had no significant impact on transcript abundance in the hippocampus of either

$\leftarrow$

subtilis, shown in the same regions. Inset regions are shown at higher magnification; scale bars: 100 and $10 \mu \mathrm{m}$, respectively. $\boldsymbol{H}$, Representative images of neocortical IEG expression after $6 \mathrm{~h}$ of ad libitum sleep ( $n=5$ mice) or SD ( $n=6$ mice). Inset regions are shown at higher magnification on right. Scale bars: $100 \mu \mathrm{m}$ (images) and $10 \mu \mathrm{m}$ (insets). I, Six-hour SD significantly increased Arc expression among non-Pvalb + cells (blue) across neocortex as a whole, and within layers $2 / 3,4$, and 5 , and increased (fos and Homer $1 a$ expression in layers 4 and 5. J, Six-hour SD significantly increased (fos expression among Pvalb + cells (magenta) in layer 2/3; no other significant changes were observed. $\boldsymbol{K}$, When analysis was restricted to IEG + Pvalb + cells (magenta, box pattern), SD significantly increased Homer $1 a$ levels among Homer $1 a+$ Pvalb + cells in whole cortex; no other significant changes were observed. Violin plots show distribution of values for individual mice; ${ }^{*} p<0.05$, ${ }^{* *} p<0.01$, Holm-Sidak post hoc test versus sleep. 
A
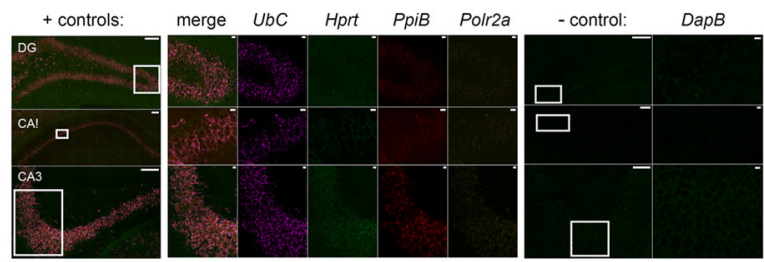

B
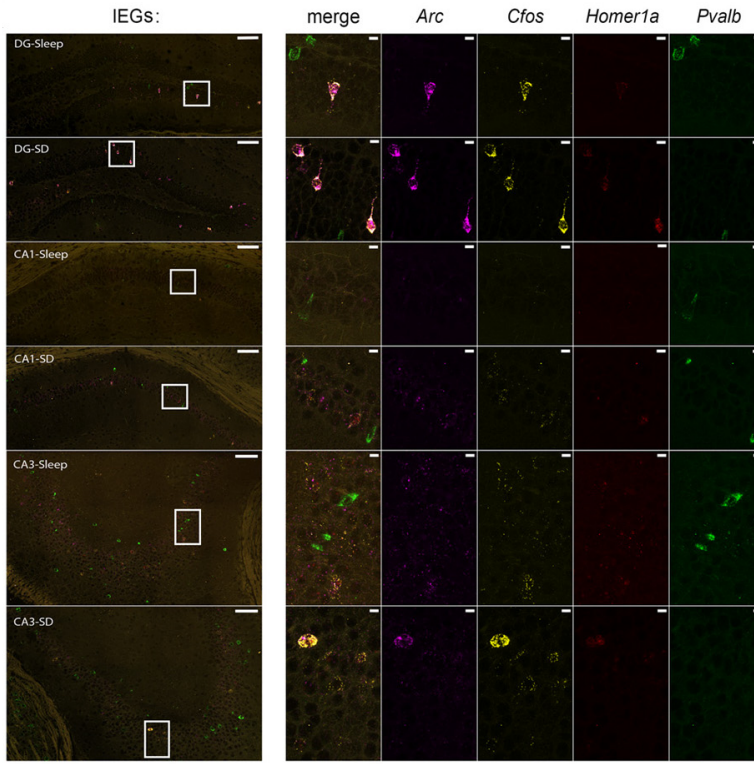

C
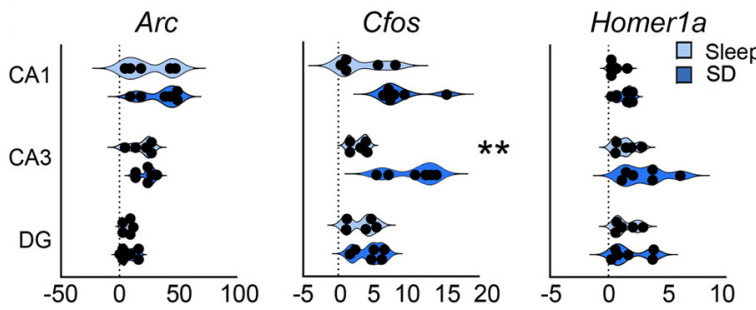

D
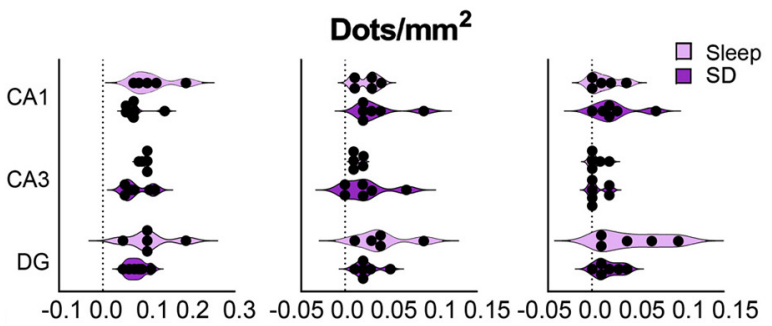

E
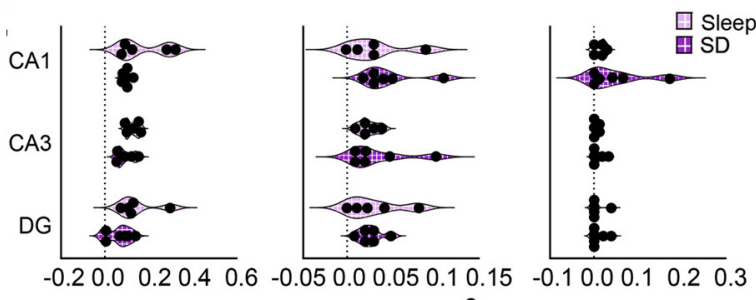

Dots $/ \mu \mathrm{m}^{2}$

Figure 4. Cell type-specific and region-specific changes in hippocampal IEG expression after SD. A, Representative in situ images showing DG, CA1, and CA3 riboprobe labeling for + control and - control genes, as in Figure $3 \mathrm{G}$. Inset regions are shown at higher magnification; scale bars: 100 and $10 \mu \mathrm{m}$, respectively. $\boldsymbol{B}$, Representative images of IEG expression after $6 \mathrm{~h}$ of ad libitum sleep ( $n=5$ mice) or SD ( $n=6$ mice). In situ hybridization is shown for Arc (magenta), Cfos (yellow), Homer1a (red), and Pvalb (green). Inset regions are shown at higher magnification on right. Scale bars: $100 \mu \mathrm{m}$ (images) and $10 \mu \mathrm{m}$ (insets). C, Six-hour SD significantly increased Cfos expression among non-Pvalb + (blue) cells in CA3; no other population. Longer-duration $(6 \mathrm{~h}) \mathrm{SD}$ resulted in no further changes in neocortical transcript abundance (with Per2 levels tending to remain elevated in both Camk2a ${ }^{+}$neurons and $\mathrm{PV}^{+}$ interneurons). Within the hippocampus, 6-h SD significantly altered abundance of ribosome-associated Per2, Cry1, and Cry2 transcripts in Camk2a ${ }^{+}$neurons (increasing Per2 and Cry1, decreasing Cry2), while having no significant effect on transcript abundance in $\mathrm{PV}^{+}$interneurons.

We also quantified (after SD vs sleep) the abundance of ribosome-associated mRNAs encoding other cellular timekeeping components: Rev-Erb $\alpha, D b p$, Ted, Nfill, and Decl (Fig. 6C). We found significant heterogeneity in how these auxiliary clock genes responded to SD in different cell types and regions. None of the transcripts were significantly altered in either cell type in the hippocampus, with either 3- or 6-h SD. However, within the neocortex, both 3- and 6-h SD significantly increased cortical Nfil3 and Dec1 abundance in $\mathrm{PV}^{+}$interneurons. While these transcripts were not significantly altered in neocortical Camk $2 \mathrm{a}^{+}$ neurons, 6-h SD significantly decreased Rev-Erb $\alpha$ expression in Camk2a ${ }^{+}$neocortical neurons. Together, these data suggest that mRNAs associated with cellular timekeeping are differentially affected, based on brain region and cell type.

\section{Discussion}

Here, using TRAP, we have identified SD-driven molecular changes unique to specific cell populations in hippocampus and neocortex. Numerous studies have used transcriptome (Cirelli et al., 2004; Vecsey et al., 2012) or proteome (Poirrier et al., 2008; Noya et al., 2019) profiling of these structures following sleep versus $\mathrm{SD}$ as a way of clarifying the functions of sleep in the brain. We find that comparing across structures, there are large differences in SD effects on ribosome-associated transcripts. For example, while even brief $(3 \mathrm{~h}) \mathrm{SD}$ increases abundance of plasticity-mediating transcripts in neocortical Camk2a ${ }^{+}$neurons and $\mathrm{PV}^{+}$interneurons (Fig. 2D), few of these transcripts are altered in hippocampus even after longer SD. This is particularly true for hippocampal $\mathrm{PV}^{+}$interneurons, for which none of the transcripts are significantly altered by SD SD-driven changes in abundance for IEG transcription regulators follow a similar pattern (Fig. 2E), with hippocampal $\mathrm{PV}^{+}$interneurons in particular being refractory to SD. Our in situ analysis of mRNA abundance in Pvalb+ and Pvalb- neurons (Figs. 3-5) is consistent with this interpretation, and suggests that even within neocortex, SD-driven changes in these transcripts' abundance are relatively modest in $\mathrm{PV}^{+}$interneurons.

Critically, our TRAP data and in situ hybridization data generally suggested similar effects of SD on mRNA abundance (i.e., comparing Figs. 2 and 3,4). Thus, in general, while our current TRAP findings cannot differentiate SD-mediated transcriptional and translational effects, a parsimonious interpretation is that SD differentially drives transcription of many genes in excitatory and inhibitory neurons. However, for some mRNA species, SDdriven increases in IEG expression for a given cell type and structure were observed using TRAP, but were not seen with in situ. At present, we cannot rule out technical differences (e.g., lower sensitivity for transcript detection using in situ compared with

significant changes observed. $\boldsymbol{D}, \boldsymbol{E}$, No significant changes were observed within $D G$, CA3, or CA1 in Pvalb + cells (magenta; $\boldsymbol{D}$ ) or IEG + Pvalb + cells (magenta, box pattern; $\boldsymbol{E}$ ). Violin plots show distribution of individual subjects; ${ }^{* *} p<0.01$, Holm-Sidak post hoc test versus sleep. 
A

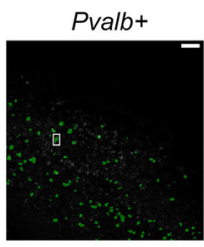

B

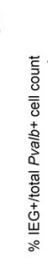
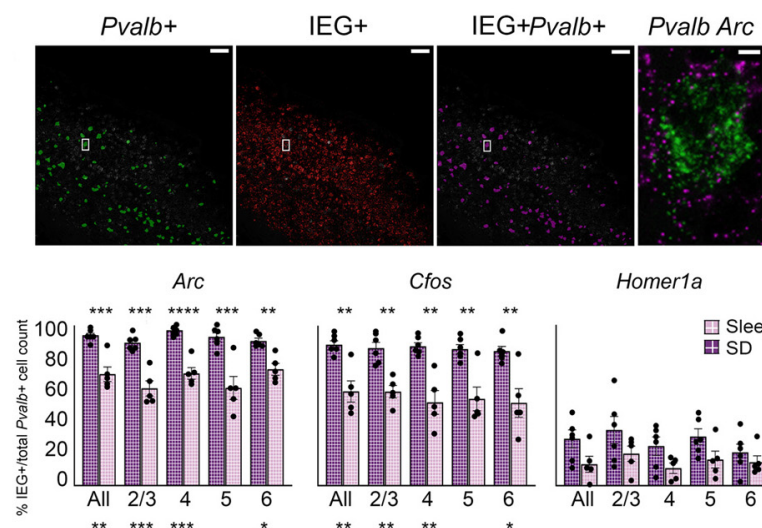

Cfos

Homer1a
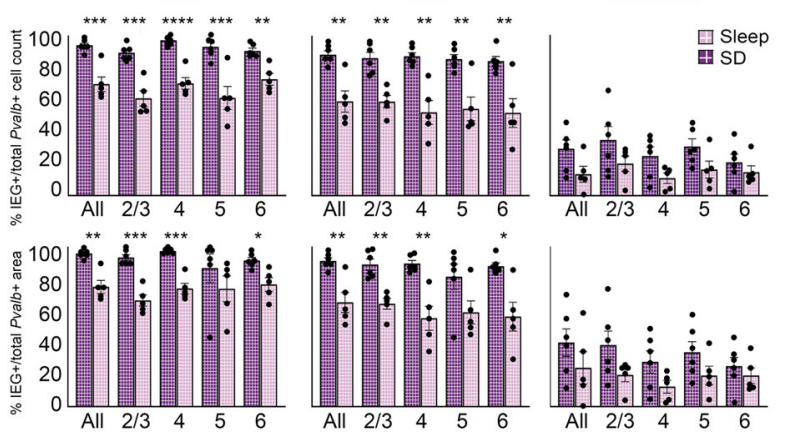

C
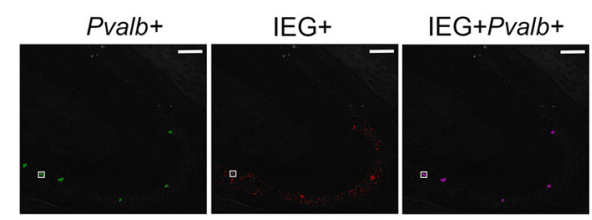

Pvalb Arc

D
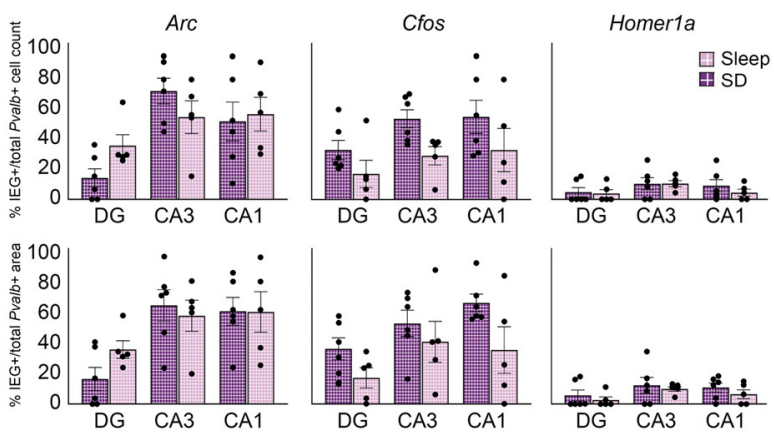

E

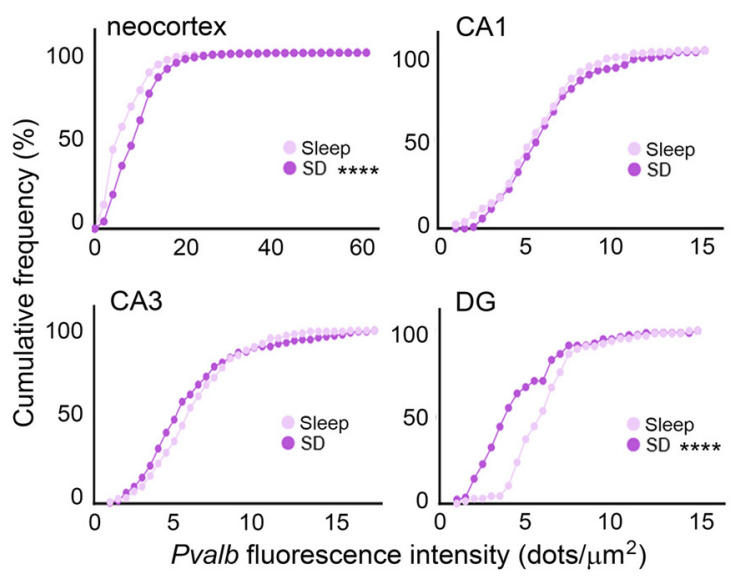

Figure 5. SD increases the proportion of IEG + Pvalb + interneurons and Pvalb expression in neocortex but not hippocampus. $\boldsymbol{A}$, An automated protocol identified Pvalb (green) and IEG (red) in situ fluorescence in neocortex; cells with overlapping fluorescence were marked as IEG + Pvalb + (magenta). Inset, In situ fluorescence for Pvalb (green) and Arc (magenta) in a single neuron, shown at right. Total IEG + Pvalb + area was then calculated as the proportion of total Pvalb + area. Scale bars: $100 \mu \mathrm{m}$ (images) and $5 \mu \mathrm{m}$ (insets). $\boldsymbol{B}$, Six-hour SD significantly increased the proportion (top row) and area (bottom row) of Pvalb + cells expressing Arc or Cfos, but not Homer1a, across most neocortical layers. Values indicate mean \pm SEM. $\boldsymbol{C}$, The same method shown in $\boldsymbol{A}$ identified IEG + Pvalb + cells within hippocampal subregions DG, CA1, and CA3. Area CA3 shown; scale bars: $100 \mu \mathrm{m}$ (images) and
TRAP) as an underlying cause of this discrepancy. Another possibility is that the specific brain areas measured using in situ (dorsal hippocampus and somatosensory and posterior parietal cortex) are selectively less sensitive to SD than other hippocampal and neocortical regions (which would contribute only to TRAP-based profiling). However, this seems unlikely, as sensory input during SD would likely drive activity higher in those particular areas (Havekes and Aton, 2020). A final, highly speculative possibility is that SD may differentially affect transcription and translation rates of specific mRNAs in some brain areas and cell types. For example, if transcription rates were unaffected, but mRNAs differentially associated with ribosomes, this could lead to differences between TRAP and in situ results we see after SD for IEGs in neocortical $\mathrm{PV}^{+}$interneurons, or for most hippocampal neurons.

While IEGs are generally assumed to reflect specific patterns of recent neuronal activity (Tyssowski and Gray, 2019), there are brain region-specific and microcircuit-specific differences in IEG expression which reflect neurons' network connectivity patterns (Tyssowski et al., 2018; Gonzales et al., 2020). Moreover, IEG expression in $\mathrm{PV}^{+}$interneurons is regulated by distinct cellular pathways and is differentially gated by neuronal activation (Cohen et al., 2016). Indeed, some studies have failed to detect IEGs in $\mathrm{PV}^{+}$interneurons altogether (Vazdarjanova et al., 2006; Imamura et al., 2011), and our present results showing relatively low expression in the Pvalb+ interneuron population (Figs. 3-5). However, insofar as abundance of all of these transcripts is likely regulated by neuronal activity to some degree (Donato et al., 2013; Yap and Greenberg, 2018), our present data support two broad conclusions. First, while SD seems to increase neuronal activation (and IEG expression) across neocortex, these effects are less pronounced in the hippocampus. Second, $\mathrm{PV}^{+}$interneuron activity (and IEG expression) may vary less as a function of SD than Camk2a ${ }^{+}$neuron activity.

The former conclusion has major implications for the field of learning and memory, where pronounced and selective effects of sleep disruption on hippocampal processes (e.g., episodic and spatial memory consolidation) have been well described (Saletin and Walker, 2012; Havekes and Abel, 2017; Delorme et al., 2019; Puentes-Mestril et al., 2019). Human brain imaging has shown that SD can lead to decreased capacity for hippocampal circuit activation during memory encoding or recall (Yoo et al., 2007). In hippocampal structures such as the DG and CA1, available data suggest that both markers of neuronal activity and synaptic plasticity are disrupted after SD (Havekes et al., 2016; Tudor et al., 2016; Ognjanovski et al., 2018; Delorme et al., 2019; Raven et al., 2019). Our present data largely confirm these findings, and suggest that particularly in dorsal hippocampal DG and CA1 (Fig. 4), there is little evidence of neuronal activity levels (and IEG expression) increasing across a period of SD. Indeed, we find that DG neurons show decreased Pvalb expression after SD, while neocortical neurons simultaneously show increased

\section{$\leftarrow$}

$5 \mu \mathrm{m}$ (insets). $\boldsymbol{D}$, SD had no effect on the proportion (top row) or area (bottom row) of Pvalb + hippocampal cells expressing Arc, Cfos, or Homer1a, Values indicate mean \pm SEM. $\boldsymbol{E}$, Cumulative frequency distributions showing the impact of 6-h SD on Pvalb fluorescence intensity in Pvalb + cells of the neocortex and hippocampus. SD significantly increased mean fluorescence intensity of Pvalb within Pvalb + cells of the neocortex. SD did not significantly alter Pvalb fluorescence intensity among Pvalb + cells in CA3 or CA1 but significantly decreased mean Pvalb fluorescence intensity within the DG while having no significant effect on $(\boldsymbol{B})$ CA1 or (C) CA3 intensity. ${ }^{*} p<0.05,{ }^{* *} p<0.01,{ }^{* * *} p<0.001$, ${ }^{* * * *} p<0.0001$, Holm-Sidak post hoc test versus sleep. 

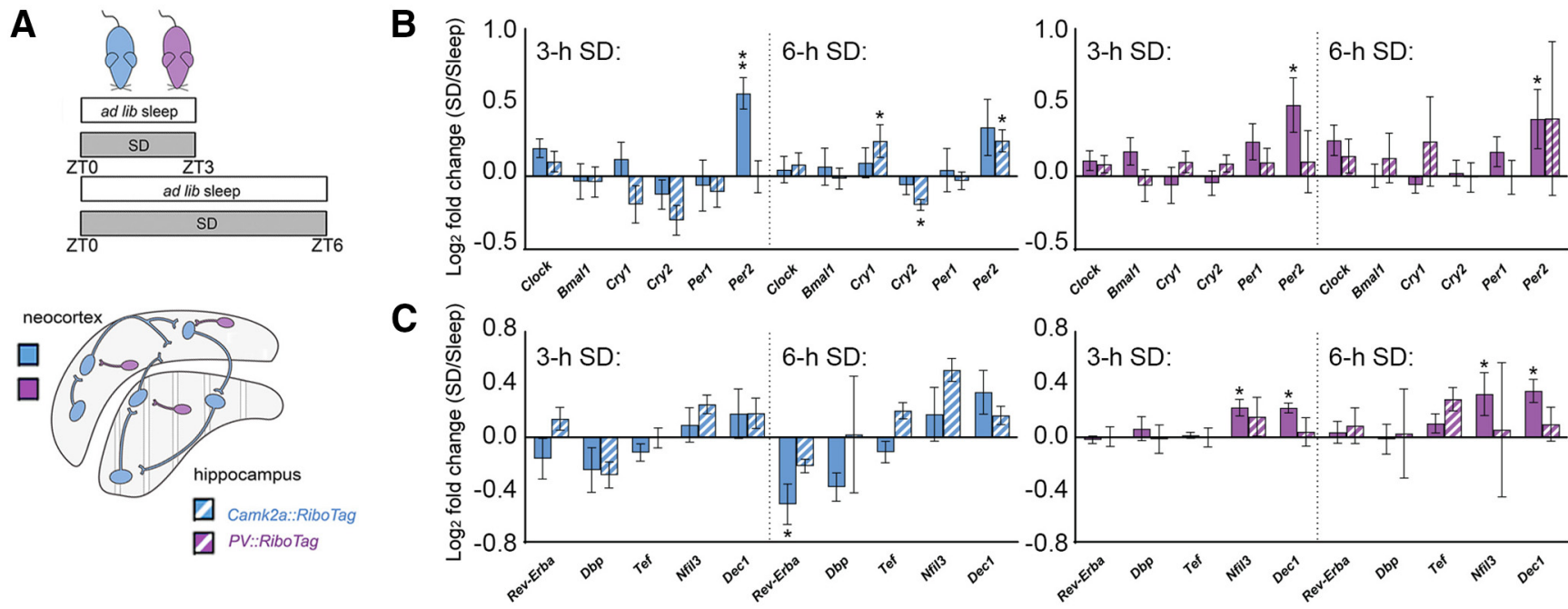

Figure 6. SD alters ribosome-associated transcripts encoding core clock genes and circadian clock modifiers in a cell type-specific and region-specific manner. $A$, Three- and 6-h SD effects on ribosome-associated transcript abundance were quantified separately from neocortex and hippocampus from Camk2a::RiboTag (blue) and PV::RiboTag (violet) transgenic mice. B, Three-hour SD significantly increased Per2 abundance on ribosomes in Camk2a ${ }^{+}$(blue) and PV ${ }^{+}$(magenta) neocortical neurons; no significant changes in core clock transcripts were observed in hippocampal neurons. After 6-h SD, Per2 abundance remained significantly elevated in neocortical PV ${ }^{+}$interneurons. Ribosome-associated Cry1, Cry2, and Per2 were all altered after 6-h SD in the hippocampal Camk2a ${ }^{+}$neuron population. No significant change observed among $\mathrm{PV}^{+}$interneurons. C, Three-hour SD had no significant effect on ribosome-associated circadian clock modifier transcripts among Camk2a ${ }^{+}$(blue) neurons in neocortex, but increased Nfil3 and Dec1 expression among neocortical PV ${ }^{+}$interneurons (magenta). Six-hour SD significantly decreased Rev-Erb $\alpha$ abundance on ribosomes in Camk2a ${ }^{+}$neocortical neurons. No transcripts were significantly altered by SD in either neuron population in hippocampus. Transcript level changes are presented as a $\log _{2}$ fold change between SD and ad libitum sleep mice. Values indicate mean \pm SEM with propagated error; ${ }^{*} p<0.05,{ }^{* *} p<0.01$, Holm-Sidak post hoc test versus sleep.

expression (Fig. 5). Critically, Pvalb expression levels have been shown to correlate with both $\mathrm{PV}^{+}$interneuron activity level and the relative amounts of excitatory to inhibitory input $\mathrm{PV}^{+}$interneurons receive (Donato et al., 2013). With this fact in mind, it is plausible that $\mathrm{SD}$ increases excitatory input to $\mathrm{PV}^{+}$interneurons in neocortex, while simultaneously decreasing excitatory input to $\mathrm{PV}^{+}$interneurons in DG. Such an effect of SD on the DG parallels our recent work showing differential effects of SD on another activity marker, Arc, in DG versus neocortex, and suggests that SD may have a uniquely disruptive effect on network activity in DG.

The latter conclusion also has important implications for maintenance of E-I balance during SD. Recent data suggest that E-I balance normally varies over the course of the day, in a sleepdependent manner (Bridi et al., 2020). Furthermore, prior evidence from both whole-tissue transcriptome profiling and immunohistochemistry has suggested that SD may differentially affect connections from excitatory to inhibitory neurons (and vice versa) in structures like the neocortex (Del Cid-Pellitero et al., 2017; Puentes-Mestril and Aton, 2017). Because sleep loss is one of the major risk factors for triggering seizure onset in epilepsy (Frucht et al., 2000; Lawn et al., 2014), an underlying mechanism might be differential activation of, or plasticity in, interneurons versus principal neurons with SD. Interactions between $\mathrm{PV}^{+}$interneurons and principal neurons are particularly important in both regulation of attention (Aton, 2013) and in generating network oscillations important for memory consolidation (Ognjanovski et al., 2017, 2018). Insofar as SD may disrupt both attention and memory consolidation, differential effects on activity of $\mathrm{PV}^{+}$and Camk2a ${ }^{+}$neurons in the hippocampus and neocortex may be an important underlying mechanism.

Because many of the transcripts quantified here (e.g., Arc, Homer1a, Narp, and Bdnf) play a critical role in activity-regulated synaptic plasticity, the fact that their abundance in Camk2 + and $\mathrm{PV}^{+}$neurons is differentially altered by $\mathrm{SD}$ also has intriguing implications. For example, it suggests that SD could lead to long-lasting changes in the E-I balance and information processing capacity of neocortical and hippocampal circuits. This may be a plausible mechanism for some of the reported longer-lasting brain metabolic (Wu et al., 2006) and cognitive (Dinges et al., 1997; Belenky et al., 2003; Chai et al., 2020) effects of SD (i.e., those that do not normalize with recovery sleep).

Alterations in brain clock gene expression with SD has been widely reported (Wisor et al., 2002, 2008; Franken et al., 2007; Mongrain et al., 2011; Bolsius et al., 2021). Along with transcripts such as Homerla (Maret et al., 2007; Zhu et al., 2020), SD-driven increases in clock transcripts such as Per2 are hypothesized to (1) act as an immediate-early gene response (similar to increases in Arc and Cfos; Balsalobre et al., 1998; Kuhlman et al., 2003; Lee et al., 2010) and (2) play a role in homeostatic aspects of sleep regulation (Franken et al., 2007; Mang and Franken, 2015). Our data suggest that similar to plasticity-regulating transcripts (including Homer 1a), SD-mediated changes in clock gene transcripts on ribosomes are cell type specific and brain region specific (Fig. 6). For example, while Per2 increases on both Camk2a $\mathrm{a}^{+}$and $\mathrm{PV}^{+}$ neocortical neuron-derived ribosomes with as little as 3-h SD, no clock gene transcripts are altered in the hippocampus with 3-h $\mathrm{SD}$. Another example is Rev-erb $\alpha$, which is significantly reduced after 6-h SD, but only in neocortical Camk2a ${ }^{+}$neurons. An interesting and important issue, raised by our findings, is that SD-driven changes in particular core clock transcripts' abundance do not move in the same direction, as they normally would during a 24 h cycle (e.g., Cry1, Cry2, and Per2). This suggests that SD-driven changes in these transcripts are not likely driven by canonical E-box elements, consistent with recent findings (Mongrain et al., 2011). However, because changes in these transcripts may have numerous downstream effects on transcription of other clock-control genes (Schmutz et al., 2010; Chiou et al., 2016), these SD-driven changes may have even more numerous downstream effects that changes in plasticity effectors' 
transcripts. Future studies will be needed to quantify longer-term cell type-specific changes to physiology and structure initiated during SD, and the molecular events responsible for these changes.

Together, our data suggest that effects of SD on plasticity, timekeeping, and homeostatic regulation of brain circuitry is heterogeneous, and likely involves subtle modifications to microcircuits (e.g., those in hippocampal subregions and neocortical layers) critical for appropriate brain function.

\section{References}

ACDBio (2017) Guideline on how to quantify RNAscope ${ }^{\otimes}$ Fluorescent Assay Results. Advanced Cell Diagnostics; Newark, CA.

Andersen CL, Jensen JL, Ørntoft TF (2004) Normalization of real-time quantitative RT-PCR data: a model based variance estimation approach to identify genes suited for normalization - applied to bladder- and coloncancer data-sets. Cancer Res 64:5245-5250.

Aton SJ (2013) Set and setting: how behavioral state regulates sensory function and plasticity. Neurobiol Learn Mem 106:1-10.

Aton SJ, Broussard C, Dumoulin M, Seibt J, Watson A, Coleman T, Frank MG (2013) Visual experience and subsequent sleep induce sequential plastic changes in putative inhibitory and excitatory cortical neurons. Proc Natl Acad Sci USA 110:3101-3106.

Balsalobre A, Damiola F, Schibler U (1998) A serum shock induces circadian gene expression in mammalian tissue culture cells. Cell 93:929-937.

Belenky G, Wesensten NJ, Thorne DR, Thomas ML, Sing HC, Redmond DP, Russo MB, Balkin TJ (2003) Patterns of performance degradation and restoration during sleep restriction and subsequent recovery: a sleep dose-response study. J Sleep Res 12:1-12.

Bolsius Y, Zurbriggen MD, Kim JK, Kas MJ, Meerlo P, Aton SJ, Havekes R (2021) The role of clock genes in sleep, stress, and memory. Biochem Pharmacol. Advance online publication. Retrieved Feb 27, 2021. doi: 10.1016/j.bcp.2021.114493.

Bridi MCD, Zong FJ, Min X, Luo N, Tran T, Qiu J, Severin D, Zhang XT, Wang G, Zhu ZJ, He KW, Kirkwood A (2020) Daily oscillation of the excitation-inhibition balance in visual cortical circuits. Neuron 105:621629.

Chai Y, Fang Z, Yang FN, Xu S, Deng Y, Raine A, Wang J, Yu M, Basner M, Goel N, Kim JJ, Wolk DA, Detre JA, Dinges DF, Rao H (2020) Two nights of recovery sleep restores hippocampal connectivity but not episodic memory after total sleep deprivation. Sci Rep 10:8774.

Chiou YY, Yang Y, Rashid N, Ye R, Selby CP, Sancar A (2016) Mammalian Period represses and de-represses transcription by displacing CLOCKBMAL1 from promoters in a cryptochrome-dependent manner. Proc Natl Acad Sci USA 113:E6072-E6079.

Cirelli C, Tononi G (2000) Differential expression of plasticity-related genes in waking and sleep and their regulation by the noradrenergic system. J Neurosci 20:9187-9194.

Cirelli C, Pompeiano M, Tononi G (1996) Neuronal gene expression in the waking state: a role for the locus coeruleus. Science 274:1211-1215.

Cirelli C, Gutierrez CM, Tononi G (2004) Extensive and divergent effects of sleep and wakefulness on brain gene expression. Neuron 41:35-43.

Clawson BC, Durkin J, Suresh AK, Pickup EJ, Broussard CG, Aton SJ (2018) Sleep promotes, and sleep loss inhibits, selective changes in firing rate, response properties and functional connectivity of primary visual cortex neurons. Front Syst Neurosci 12:40.

Clawson BC, Pickup EJ, Ensing A, Geneseo L, Shaver J, Gonzalez-Amoretti J, Zhao M, York AK, Kuhn FR, Swift K, Martinez JD, Wang L, Jiang S, Aton SJ (2021) Causal role for sleep-dependent reactivation of learningactivated sensory ensembles for fear memory consolidation. Nat Commun 12:1200.

Cohen SM, Ma H, Kuchibhotla KV, Watson BO, Buzsáki G, Froemke RC, Tsien RW (2016) Excitation-transcription coupling in parvalbumin-positive interneurons employs a novel CaM kinase-dependent pathway distinct from excitatory neurons. Neuron 90:292-307.

Del Cid-Pellitero E, Plavski A, Mainville L, Jones BE (2017) Homeostatic changes in GABA and glutamate receptors on excitatory cortical neurons during sleep deprivation and recovery. Front Syst Neurosci 11:17.

Delorme JE, Kodoth V, Aton SJ (2019) Sleep loss disrupts Arc expression in dentate gyrus neurons. Neurobiol Learn Mem 160:73-82. de Vivo L, Bellesi M, Marshall W, Bushong EA, Ellisman H, Tononi G, Cirelli C (2017) Ultrastructural evidence for synaptic scaling across the wake/sleep cycle. Science 355:507-510.

Dinges DF, Pack F, Williams K, Gillen KA, Powell JW, Ott GE, Aptowicz C, Pack AI (1997) Cumulative sleepiness, mood disturbance, and psychomotor vigilance performance decrements during a week of sleep restricted to 4-5 hours per night. Sleep 20:267-277.

Donato F, Rompani SB, Caroni P (2013) Parvalbumin-expressing basket-cell network plasticity induced by experience regulates adult learning. Nature 504:272-276.

Durkin J, Aton SJ (2016) Sleep-dependent potentiation in the visual system is at odds with the synaptic homeostasis hypothesis. Sleep 39:155-159.

Durkin J, Suresh AK, Colbath J, Broussard C, Wu J, Zochowski M, Aton SJ (2017) Cortically coordinated NREM thalamocortical oscillations play an essential, instructive role in visual system plasticity. Proc Natl Acad Sci USA 114:10485-10490.

Franken P, Thomason R, Heller HC, O'Hara BF (2007) A non-circadian role for clock-genes in sleep homeostasis:a strain comparison. BMC Neurosci $8: 87$

Frucht MM, Quigg M, Schwaner C, Fountain NB (2000) Distribution of seizure precipitants among epilepsy syndromes. Epilepsia 41:1534-1539.

Gerashchenko D, Wisor JP, Burns D, Reh RK, Shiromani PJ, Sakurai T, de la Iglesia HO, Kilduff TS (2008) Identification of a population of sleepactive cerebral cortex neurons. Proc Natl Acad Sci USA 105:1022710232 .

Gonzales BJ, Mukherjee D, Ashwal-Fluss R, Loewenstein Y, Citri A (2020) Subregion-specific rules govern the distribution of neuronal immediateearly gene induction. Proc Natl Acad Sci USA 117:23304-23310.

Havekes R, Abel T (2017) The tired hippocampus: the molecular impact of sleep deprivation on hippocampal function. Curr Opin Neurobiol 44:1319.

Havekes R, Aton SJ (2020) Impacts of sleep loss versus waking experience on brain plasticity: parallel or orthogonal? Trends Neurosci 43:385-393.

Havekes R, Park AJ, Tudor JC, Luczak VG, Hansen RT, Ferri SL, Bruinenberg VM, Poplawski SG, Day JP, Aton SJ, Radwańska K, Meerlo P, Houslay MD, Baillie GS, Abel T (2016) Sleep deprivation causes memory deficits by negatively impacting neuronal connectivity in hippocampal area CA1. Elife 5:e13424.

Hoekstra MMB, Emmenegger Y, Hubbard J, Franken P (2019) Cold-inducible RNA-binding protein (CIRBP) adjusts clock-gene expression and REM-sleep recovery following sleep deprivation. Elife 8:e43400.

Imamura N, Nonaka A, Yamamoto H, Matsuki N, Nomura H (2011) Experience-dependent Homerla expression in excitatory and inhibitory neurons. Neuroreport 22:353-357.

Jiang Y, Gong NN, Hu XS, Ni MJ, Pasi R, Matsunami H (2015) Molecular profiling of activated olfactory neurons identifies odorant receptors for odors in vivo. Nat Neurosci 18:1446-1454.

Kuhlman SJ, Silver R, Le Sauter J, Bult-Ito A, McMahon DG (2003) Phase resetting light pulses induce Per1 and persistent spike activity in a subpopulation of biological clock neurons. J Neurosci 23:1441-1450.

Kuhlman SJ, Olivas ND, Tring E, Ikrar T, Xu X, Trachtenberg JT (2013) A disinhibitory microcircuit initiates critical period plasticity in visual cortex. Nature 501:543-546.

Lawn N, Lieblich S, Lee J, Dunne J (2014) Are seizures in the setting of sleep deprivation provoked? Epilepsy Behav 33:122-125.

Lee Y, Lee J, Kwon I, Nakajima Y, Ohmiya Y, Son GH, Lee KH, Kim K (2010) Coactivation of the CLOCK-BMAL1 complex by CBP mediates resetting of the circadian clock. J Cell Sci 123:3547-3557.

Mackiewicz M, Shockley KR, Romer MA, Galante RJ, Zimmerman JE, Naidoo N, Baldwin DA, Jensen ST, Churchill GA, Pack AI (2007) Macromolecule biosynthesis - a key function of sleep. Physiol Genomics 31:441-457.

Mang GM, Franken P (2015) Genetic dissection of sleep homeostasis. Curr Top Behav Neurosci 25:25-53.

Maret S, Dorsaz S, Gurcel L, Pradervand S, Petit B, Pfister C, Hagenbuchle O, O'Hara BF, Franken P, Tafti M (2007) Homerla is a core brain molecular correlate of sleep loss. Proc Natl Acad Sci USA 104:20090-20095.

McDermott CM, LaHoste GJ, Chen C, Musto A, Bazan NG, Magee JC (2003) Sleep deprivation causes behavioral, synaptic, and membrane excitability alterations in hippocampal neurons. J Neurosci 23:9687-9695.

Mednick S, Nakayama K, Stickgold R (2003) Sleep-dependent learning: a nap is as good as a night. Nat Neurosci 6:697-698. 
Mikhail C, Vaucher A, Jimenez S, Tafti M (2017) ERK signaling pathway regulates sleep duration through activity-induced gene expression during wakefulness. Sci Signal 10:eaai9219.

Mongrain V, La Spada F, Curie T, Franken P (2011) Sleep loss reduces the DNA-binding of BMAL1, CLOCK, and NPAS2 to specific clock genes in the mouse cerebral cortex. PLoS One 6:e26622.

Noya SB, Colameo D, Brüning F, Spinnler A, Mircsof D, Opitz L, Mann M, Tyagarajan SK, Robles MS, Brown SA (2019) The forebrain synaptic transcriptome is organized by clocks but its proteome is driven by sleep. Science 366:eaav2642.

Ognjanovski N, Schaeffer S, Mofakham S, Wu J, Maruyama D, Zochowski M, Aton SJ (2017) Parvalbumin-expressing interneurons coordinate hippocampal network dynamics required for memory consolidation. Nat Commun 8:15039.

Ognjanovski N, Broussard C, Zochowski M, Aton SJ (2018) Hippocampal network oscillations rescue memory consolidation deficits caused by sleep loss. Cereb Cortex 28:3711-3723.

Poirrier JE, Guillonneau F, Renaut J, Sergeant K, Luxen A, Maquet P, Leprince P (2008) Proteomic changes in rat hippocampus and adrenals following short-term sleep deprivation. Proteome Sci 6:14.

Puentes-Mestril C, Aton SJ (2017) Linking network activity to synaptic plasticity during sleep: hypotheses and recent data. Front Neural Circuits 11:61.

Puentes-Mestril C, Roach J, Niethard N, Zochowski M, Aton SJ (2019) How rhythms of the sleeping brain tune memory and synaptic plasticity. Sleep 42:zsz095.

Raven F, Meerlo P, Van der Zee EA, Abel T, Havekes R (2019) A brief period of sleep deprivation causes spine loss in the dentate gyrus of mice. Neurobiol Learn Mem 160:83-90.

Saletin JM, Walker MP (2012) Nocturnal mnemonics: sleep and hippocampal memory processing. Front Neurol 3:59.

Sanz E, Yang L, Su T, Morris DR, McKnight GS, Amieux PS (2009) Celltype-specific isolation of ribosome-associated mRNA from complex tissues. Proc Natl Acad Sci USA 106:13939-13944.

Sanz E, Bean JC, Carey DP, Quintana A, McKnight GS (2019) RiboTag: ribosomal tagging strategy to analyze cell-type-specific mRNA expression in vivo. Curr Protoc Neurosci 88:e77.

Schmutz I, Ripperger JA, Baeriswyl-Aebischer S, Albrecht U (2010) The mammalian clock component PERIOD2 coordinates circadian output by interaction with nuclear receptors. Genes Dev 24:345-357.

Shigeoka T, Jung J, Holt CE, Jung H (2018) Axon-TRAP-RiboTag: affinity purification of translated mRNAs from neuronal axons in mouse in vivo. Methods Mol Biol 1649:85-94.

Sosa JM, Huber DE, Welk B, Fraser HL (2014) Development and application of MIPAR ${ }^{\mathrm{TM}}$ : a novel software package for two- and three-dimensional microstructural characterization. Integr Mater Manuf Innov 3:123-140.

Stickgold R (2005) Sleep-dependent memory consolidation. Nature 437:1272-1278.

Terao A, Wisor JP, Peyron C, Apte-Deshpande A, Wurts SW, Edgar DM, Kilduff TS (2006) Gene expression in the rat brain during sleep deprivation and recovery sleep: an Affymetrix GeneChip(R) study. Neuroscience 137:593-605.

Tononi G, Cirelli C (2006) Sleep function and synaptic homeostasis. Sleep Med Rev 10:49-62.

Tudor JC, Davis EJ, Peixoto L, Wimmer ME, van Tilborg E, Park AJ, Poplawski SG, Chung CW, Havekes R, Huang J, Gatti E, Pierre P, Abel T (2016) Sleep deprivation impairs memory by attenuating mTORC1-dependent protein synthesis. Sci Signal 9:ra41.

Tyssowski KM, Gray JM (2019) The neuronal stimulation-transcription coupling map. Curr Opin Neurobiol 59:87-94.

Tyssowski KM, DeStefino NR, Cho JH, Dunn CJ, Poston RG, Carty CE, Jones RD, Chang SM, Romeo P, Wurzelmann MK, Ward JM, Andermann ML, Saha RN, Dudek SM, Gray JM (2018) Different neuronal activity patterns induce different gene expression programs. Neuron 98:530-546.

Vazdarjanova A, Ramirez-Amaya V, Insel N, Plummer TK, Rosi S, Chowdhury S, Mikhael D, Worley PF, Guzowski JF, Barnes CA (2006) Spatial exploration induces ARC, a plasticity-related immediate-early gene, only in calcium/calmodulin-dependent protein kinase II-positive principal excitatory and inhibitory neurons of the rat forebrain. J Comp Neurol 498:317-329.

Vecsey CG, Peixoto L, Choi JHK, Wimmer M, Jaganath D, Hernandez PJ, Blackwell J, Meda K, Park AJ, Hannenhalli S, Abel T (2012) Genomic analysis of sleep deprivation reveals translational regulation in the hippocampus. Physiol Genomics 44:981-991.

Vyazovskiy VV, Olcese U, Lazimy YM, Faraguna U, Esser SK, Williams JC, Cirelli C, Tononi G (2009) Cortical firing and sleep homeostasis. Neuron 63:865-878.

Wisor JP, O'Hara BF, Terao A, Selby CP, Kilduff TS, Sancar A, Edgar DM, Franken P (2002) A role for cryptochromes in sleep regulation. BMC Neurosci 3:20.

Wisor JP, Pasumarthi RK, Gerashchenko D, Thompson CL, Pathak S, Sancar A, Franken P, Lein ES, Kilduff TS (2008) Sleep deprivation effects on circadian clock gene expression in the cerebral cortex parallel electroencephalographic differences among mouse strains. J Neurosci 28:71937201.

Wu JC, Gillin JC, Buchsbaum MS, Chen P, Keator DB, Khosla Wu N, Darnall LA, Fallon JH, Bunney WE (2006) Frontal lobe metabolic decreases with sleep deprivation not totally reversed by recovery sleep. Neuropsychopharmacol 31:2783-2792.

Xie F, Peng X, Chen D, Lei X, Zhang B (2012) miRDeepFinder: a miRNA analysis tool for deep sequencing of plant small RNAs. Plant Mol Biol 80:75-84

Yap EL, Greenberg ME (2018) Activity-regulated transcription: bridging the gap between neural activity and behavior. Neuron 100:330-348.

Yoo SS, Hu PT, Gujar N, Jolesz FA, Walker MP (2007) A deficit in the ability to form new human memories without sleep. Nat Neurosci 10:385-392.

Zhu J, Hafycz J, Keenan BT, Guo X, Pack AI, Naidoo N (2020) Acute sleep loss upregulates the synaptic scaffolding protein, Homerla, in non-canonical sleep/wake brain regions, claustrum, piriform and cingulate cortices. Front Neurosci 14:188. 